\title{
Optimal Causal Rate-Constrained Sampling of the Wiener Process
}

\author{
Nian Guo and Victoria Kostina
}

\begin{abstract}
We consider the following communication scenario. An encoder causally observes the Wiener process and decides when and what to transmit about it. A decoder makes real-time estimation of the process using causally received codewords. We determine the causal encoding and decoding policies that jointly minimize the mean-square estimation error, under the long-term communication rate constraint of $R$ bits per second. We show that an optimal encoding policy can be implemented as a causal sampling policy followed by a causal compressing policy. We prove that the optimal encoding policy samples the Wiener process once the innovation passes either $\sqrt{\frac{1}{R}}$ or $-\sqrt{\frac{1}{R}}$, and compresses the sign of the innovation (SOI) using a 1-bit codeword. The SOI coding scheme achieves the operational distortion-rate function, which is equal to $D^{\mathrm{op}}(R)=\frac{1}{6 R}$. Surprisingly, this is significantly better than the distortion-rate tradeoff achieved in the limit of infinite delay by the best non-causal code. This is because the SOI coding scheme leverages the free timing information supplied by the zero-delay channel between the encoder and the decoder. The key to unlock that gain is the event-triggered nature of the SOI sampling policy. In contrast, the distortion-rate tradeoffs achieved with deterministic sampling policies are much worse: we prove that the causal informational distortion-rate function in that scenario is as high as $D_{\mathrm{DET}}(R)=\frac{5}{6 R}$. It is achieved by the uniform sampling policy with the sampling interval $\frac{1}{R}$. In either case, the optimal strategy is to sample the process as fast as possible and to transmit 1-bit codewords to the decoder without delay.

Index Terms-Causal lossy source coding, sequential estima-
\end{abstract} tion, sampling.

\section{INTRODUCTION}

\section{A. System Model}

Consider the system in Fig. 1. A source outputs a continuous-time standard Wiener process $\left\{W_{t}\right\}_{t=0}^{T}$, within the time horizon $[0, T]$. An encoder observes the process and decides to disclose information about it at a sequence of nondecreasing codeword-generating time stamps

$$
0 \leq \tau_{1} \leq \tau_{2} \leq \cdots \leq \tau_{N} \leq T
$$

These time stamps can be random and they can causally depend on the Wiener process. Consequently, the total number of time stamps $N$ can also be random. At time $\tau_{i}$, the encoder chooses to generate a binary codeword $U_{i}$, with a length $\ell_{i} \in \mathbb{Z}^{+}$, based on the past observed process $\left\{W_{t}\right\}_{t=0}^{\tau_{i}}$. Then, the codeword $U_{i}$ is passed through a noiseless digital channel to the decoder without delay. Upon receiving the codeword $U_{i}$ at time $\tau_{i}$, based on all the received codewords $U^{i}$ and the codeword-generating time stamps $\left\{\tau_{1}, \ldots, \tau_{i}\right\}$, the decoder updates its running estimate of the Wiener process, yielding

N. Guo and V. Kostina are with the Department of Electrical Engineering, California Institute of Technology, Pasadena, CA, 91125 USA. Email: \{nguo,vkostina\}@caltech.edu. This work was supported in part by the National Science Foundation (NSF) under grant CCF-1751356.
$\left\{\hat{W}_{t}\right\}_{t=\tau_{i}}^{T}$. The decoder updates its estimate $\left\{\hat{W}_{t}\right\}_{t=\tau_{i+1}}^{T}$ once the next codeword $U_{i+1}$ is received at $\tau_{i+1}$.

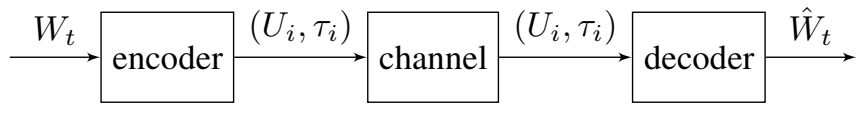

Fig. 1: System Model.

The communication between the encoder and the decoder is subject to a constraint on the long-term average transmission rate,

$$
\frac{1}{T} \mathbb{E}\left(\sum_{i=1}^{N} \ell_{i}\right) \leq R \text { (bits per sec). }
$$

The distortion is measured by the long-term mean-square error (MSE) between $W_{t}$ and $\hat{W}_{t}, 0 \leq t \leq T$,

$$
\frac{1}{T} \mathbb{E}\left(\int_{0}^{T}\left(W_{t}-\hat{W}_{t}\right)^{2} d t\right) \leq d
$$

We aim to find the jointly optimal encoding and decoding policies that achieve the best tradeoffs between the rate in (2) and the MSE in (3).

\section{B. Literature Review}

Finding sampling policies at the encoder and estimation policies at the decoder to jointly minimize the end-to-end distortion under transmission constraints falls into the realm of optimal scheduling and sequential estimation problems. These problems are often encountered in network control systems, and has attracted significant research interest with the development of robotics, the Internet of things, and the smart grid.

Åström and Bernhardsson [1] compared uniform and symmetric threshold sampling policies ${ }^{1}$ (referred to as Riemann and Lebesgue sampling, respectively) in continuous-time firstorder stochastic systems with a Wiener process disturbance, and showed that the Lebesgue sampling gives a lower distortion than the Riemann sampling under the same average sampling frequency. Imer and Başar [2] considered causal estimation of i.i.d. processes under MSE and the constraint on the total number of transmissions over a finite time horizon, and showed via dynamic programming, that the timevarying symmetric threshold sampling policy is optimal for i.i.d. Gaussian processes [2]. For causal estimation of multidimensional discrete-time Gauss-Markov processes, Cogill et al. [3] aimed to find a sampling policy that minimizes a cost function combining the average weighted MSE and the

\footnotetext{
${ }^{1}$ The symmetric threshold sampling corresponds to sampling the process if its current value exceeds or falls short of the previous sample by exactly a certain threshold.
} 
average transmission cost over the infinite horizon. Cogill et al. [3] proposed a threshold policy that transmits a sample once the absolute value of the squared error exceeds some constant, and proved that this suboptimal policy leads to a cost that is within a factor of 6 of the optimal achievable cost. Using dynamic programming and majorization theory, Lipsa and Martins [4] proved that a time-varying symmetric threshold policy and a Kalman-like filter jointly minimize a discounted cost function consisting of MSE and a communication cost, for scalar discrete-time Gauss-Markov processes over a finite time horizon. For partially observed discrete-time Gauss-Markov processes, Wu et al. [5] fixed an event-triggered policy, where the encoder transmits only if the L-infinity norm of the Mahalanobis transformation of the measurement innovation exceeds a constant, and derived both the accurate and approximate (assuming Gaussian samples) minimum MSE (MMSE) estimator to combine with that sampling policy. $\mathrm{Wu}$ et al. [5] also derived the relation between the transmission frequency and the threshold of the event-triggered policy. Rabi et al. [6] formulated the problem of causal estimation of continuous-time scalar linear diffusion processes under the constraint on the total number of transmissions over a finite time horizon as an optimal stopping time problem. Rabi et al. [6] solved the optimal stopping time problem iteratively to show that the optimal deterministic sampling policy for the Wiener process is a uniform sampling policy, and that the optimal event-triggered sampling policy is a time-varying symmetric threshold policy. Rabi et al. [6] derived a dynamic program that can be used to numerically compute the optimal sampling policy for the Ornstein-Uhlenbeck process. Nar and Başar [7] extended the optimal stopping time problem in [6] to the multidimensional Wiener process, and proved that a symmetric threshold policy remains optimal over both finite and infinite time horizons. In particular, Nar and Başar [7] showed that the optimal threshold over the infinite horizon is a constant depending on the average sampling frequency. For autogressive Markov processes driven by an i.i.d. process with unimodal and symmetric distribution, Charkravorty and Mahajan [8] used ideas from renewal theory to prove that there is no loss of optimality if one focuses on sampling policies with time-homogeneous thresholds over the infinite time horizon. Charkravorty and Mahajan [8] also proved that a symmetric threshold sampling policy together with an Kalman-like estimator achieves the distortion-transmission function, where the distortion-transmission function is defined as the minimum achievable expected average (or discounted) distortion subject to the expected average (or discounted) number of transmissions. For the same scenario as in [8], Molin and Hirche [9] proposed an iterative algorithm to show that the optimal event-triggered policy converges to a symmetric threshold policy.

In contrast to the scenarios in [1]-[9], where the communication channel is assumed to be perfect, [10]-[12] consider imperfect communication channels, such as a channel with an i.i.d. delay [10], a channel with i.i.d. Gamma noise [11], a fading channel [12], and a packet-drop channel [13]. Sun et al.
[10] proved that a symmetric threshold policy remains optimal even when the samples of the Wiener process experience an i.i.d. random transmission delay, but the threshold depends on the distribution of channel delay and is different from the one in [7]. Using dynamic programming, Gao et al. in [11] derived the optimal sampling, encoding and decoding policies for event-triggered sampling of an i.i.d. Laplacian source with subsequent transmission over a channel with a Gamma additive noise, under an average power constraint. For discrete-time first-order autogressive Markov processes considered in [8]-[9], Ren et al. [12] introduced a fading channel between the encoder and the decoder, where a successful transmission depends on both the channel gains and the transmission power, and found the optimal encoding and decoding policies that minimize an infinite horizon cost function combining the MSE and the power usage. For first-order autogressive sources considered in [8][9][12], Chakravorty and Mahajan [13] further proved that the optimal estimation policy is a Kalman-like filter and the optimal sampling policy is symmetric threshold policy when the communication channel is a packet-drop channel with Markovian states, where the packet-drop probability depends on the channel state and the transmission power chosen by the encoder.

Nayyar et al. [14] considered a scenario where the encoder relies on the energy harvested from the environment to transmit messages to the estimator, with each transmission costing 1 unit of energy, and proved that the optimal sampling strategy is a symmetric threshold policy, provided that the finitestate Markov source has symmetric and unimodal distribution and the distortion measure is either the Hamming distortion function or the power of the estimation error $|x-y|^{p}$. For the non-causal lossy source coding of a uniformly sampled Wiener process, Kipnis et al. [15] derived the trade-offs between the sampling frequency, the communication bitrate and the estimation MSE, achievable in the limit of inifinite delay.

Kofman and Braslavsky [16] designed a quantized event-triggered controller for noiseless partially observed continuous-time LTI systems with an unknown initial state to ensure asymptotic convergence of the system to the origin with zero average rate, seemingly violating the data-rate theorem. Similar to [16], the fact that sampling time stamps of eventtriggered policies carry information is also exploited in [17][20]. Pearson et al. [17] considered encoding the deterministic and possibly nonuniformly sampled states of noiseless continuous-time LTI systems into symbols in a finite alphabet with a free symbol representing the absence of transmission. For discrete-time linear systems with additive disturbances, Khina et al. [18] considered a setting where at each discretetime instant, the encoder chooses to either transmit 1 bit or transmit the free symbol, and designed an adaptive scalar quantizer with three bins using a Lloyd-Max algorithm with the quantization bin of the largest probability corresponding to the free symbol. Ling [19] designed a periodic eventtriggered quantization policy to stabilize continuous-time LTI systems subject to i.i.d. feedback dropouts, bounded network delay and bounded noise, which leads to a stabilizing rate 
that is lower than the one the data-rate theorem requires for time-triggered policies. Khojasteh et al. [20] considered sampling noiseless continuous-time LTI systems when the state estimation error exceeds an exponentially decaying function, and found that the information transmission rate required for stabilizing systems can be any positive value for small enough delays and starts to increase when the delay exceeds a critical value. Quantized event-triggered control has also been studied for continuous-time LTI systems with bounded disturbances [21], for partially-observed continuoustime LTI systems without noise [22] and with bounded noise [23], for discrete-time noiseless linear systems [24], and for partially observed continuous-time LTI systems with timevarying network delay [25]. Event-triggered control schemes to guarantee exponential stabilization were designed both for continuous-time LTI systems with bounded disturbances under a bounded rate constraint [26] and for noiseless continuoustime LTI systems under time-varying rates constraints and channel blackouts [27].

\section{Contribution}

In this paper, we adopt an information-theoretic approach to continuous-time causal estimation, by considering the optimal tradeoff between the achievable MSE and the average number of bits communicated. This is different from the models studied in [1]-[14], where communication cost is measured by the number of transmissions, and each infinite-precision transmission can carry an infinite amount of information. For communication over digital channels, a bitrate constraint, routinely considered in information theory, is more appropriate. Our setting is also different from [15] in that we do not ignore delay: our distortion at time $t$ is measured with respect to the actual value of the process at time $t$; whereas [15] permits an infinite delay, following a standard assumption in information theory. In contrast to the works [16]-[20] that do not claim or consider the optimality of the proposed event-triggered policies, we show the optimality of the SOI coding scheme for our problem setting in Section I-A.

We first show that an optimal encoding policy that achieves the operational distortion-rate function (ODRF) can be implemented as a causal sampling policy coupled with a compressing policy. Then, we prove that the optimal encoding policy is a symmetric threshold sampling policy with threshold $\pm \sqrt{\frac{1}{R}}$ and a 1-bit SOI compressor. The optimal decoding policy causally estimates the Wiener process by summing up the received innovations. This coding scheme, termed the SOI coding scheme, achieves the ODRF $D^{\mathrm{op}}(R)=\frac{1}{6 R}$.

In the SOI coding scheme, the encoder continuously tracks the process, generating a bit once the process passes the threshold. To reconstruct the process, both those bits and their time stamps are required at the decoder. In the scenario where, due to implementation constraint, the sampler is processagnostic, or the decoder has no access to timing information, one has to adopt a deterministic sampling policy. We prove that a uniform sampling policy with the sampling interval $\frac{1}{R}$ achieves the informational distortion-rate function (IDRF), which is equal to $D_{\mathrm{DET}}(R)=\frac{5}{6 R}$. To define the IDRF for the deterministic sampling policies, we change the rate constraint (2) to a directed mutual information rate constraint, which serves as an information-theoretic lower bound to (2). This is a consequence of our real-time distortion constraint. Had we allowed delay, coding gains would have been possible by, for example, jointly compressing blocks of those bits. To confirm that the IDRF is a meaningful gauge of what is achievable in the zero-delay causal compression, we implement the greedy Lloyd-Max compressor [18] to compress the innovations $W_{\tau_{i}}-\hat{W}_{\tau_{i-1}}$, and verify that the performance of the resulting scheme is close to the IDRF.

To study the tradeoffs between the sampling frequency and the rate per sample under a rate per second constraint $R$, we define operational and informational distortion-frequency-rate function (ODFRF and IDFRF). The ODFRF and the IDFRF are both minimized by the maximum sampling frequency $R$ and the minimum rate $1 \mathrm{bit} / \mathrm{sample}$, implying that sampling the process as fast as possible under the rate constraint and transmitting 1-bit codewords to the decoder without delay is optimal.

Surprisingly, the distortion achieved by the SOI coding scheme is smaller than the distortion achieved by the best noncausal codes. The reason is that in the SOI coding scheme, the encoder and the decoder know the random sampling time stamps perfectly, whereas in classical non-causal coding, this free timing information is not considered.

We also show that the SOI coding scheme with a different sampling threshold continues to be optimal when there is a random i.i.d. channel delay between the codeword-generating time and the codeword-delivery time. Finally, we show that if the decoder is allowed to wait for only the next codeword before decoding, the MSE can be further decreased.

A part of this work will be presented at the 57th Annual Allerton Conference [38]; the conference version does not contain any proofs.

\section{Paper organization}

In Section II, we define causal codes, distortion-rate and distortion-frequency-rate functions. In Section III, we state the main results of this paper, including the optimal causal sampling and compressing policies and the tradeoffs between the sampling frequency and the rate per sample. In Section IV, we show the proof of the main results. In Section V, we discuss the distortion-rate tradeoffs when delays are allowed at both the encoder and the decoder, at the decoder only, and at the communication channel.

\section{E. Notations}

We denote by $\left\{W_{t}\right\}_{t=\tau_{i}}^{\tau_{i+1}}$ and $\left\{W_{t}\right\}_{\tau_{i}<t<\tau_{i+1}}$ the parts of the Wiener process within time intervals $\left[\tau_{i}, \tau_{i+1}\right]$, and $\left(\tau_{i}, \tau_{i+1}\right)$, respectively. For $M \in \mathbb{Z}^{+},[M] \triangleq\{1, \ldots, M\}$. For a possibly infinite sequence $x=\left\{x_{1}, x_{2}, \ldots\right\}$, we write $x^{i}=\left\{x_{1}, x_{2}, \ldots, x_{i}\right\}$ to denote the vector of its first $i$ elements. 


\section{Distortion-RATE FUNCTIONS}

In this section, we define the operational and the informational causal distortion-rate functions, and we show that an optimal encoder can be separated into a sampler followed by a compressor.

\section{A. Encoding and decoding policies}

The standard Wiener process is defined as follows.

Definition 1. (standard Wiener process, e.g. [31]) A standard Wiener process $\left\{W_{t}\right\}_{t \geq 0}$ is a stochastic process characterized by the following three properties:

(i) time-homogeneity: for all non-negative $s$ and $t, W_{s}$ and $W_{s+t}-W_{t}$ have the same distribution $\left(W_{0}=0\right)$;

(ii) independent increments: $W_{t_{i}}-W_{s_{i}}(i \geq 1)$ are independent whenever the intervals $\left(s_{i}, t_{i}\right]$ are disjoint;

(iii) $W_{t}$ follows the Gaussian distribution $\mathcal{N}(0, t)$.

Throughout, we assume that both encoder and decoder know the initial state $W_{0}=0$ at $\tau_{0}=0$.

Next, we formally define the encoding and decoding policies $^{2}$. Denote the set of continuous functions on the time interval $[0, t]$ by $\mathcal{C}_{[0, t]}$. Define the Wiener process stopped at a stopping time $\tau$ (e.g. [33, Eq. 3.9]) as:

$$
W_{t}(\tau)= \begin{cases}W_{t} & \text { if } t \leq \tau \\ W_{\tau} & \text { if } t>\tau\end{cases}
$$

Definition 2. (An $(R, d, T)$ causal code) An $(R, d, T)$ causal code for the Wiener process $\left\{W_{t}\right\}_{t=0}^{T}$ is a pair of encoding and decoding policies defined as follows.

The encoding policy consists of

(i) the causal sampling policy $\pi_{T}=\left\{\tau_{1}, \tau_{2}, \ldots\right\}$ that decides the codeword-generating time stamps in (1) that are stopping times of the filtration $\sigma\left(\left\{W_{t}\right\}_{t=0}^{T}\right)$, and

(ii) the compressing policy $f_{T}=\left\{f_{1}, f_{2}, \ldots\right\}^{3}$,

$$
\mathrm{f}_{i}: \mathcal{C}_{[0, T]} \rightarrow\left[2^{\ell i}\right] .
$$

The codeword generated at time $\tau_{i}$ is $U_{i}=\mathrm{f}_{i}\left(\left\{W_{t}\left(\tau_{i}\right)\right\}_{t=0}^{T}\right)$. The codewords' lengths must satisfy the long-term average rate constraint (2).

The decoding policy causally maps the received codewords and the codeword-generating time stamps to a continuous-time process estimate $\left\{\hat{W}_{t}\right\}_{t=0}^{T}$ using

$$
\hat{W}_{t}=\hat{W}_{\tau_{i}} \triangleq \mathbb{E}\left(W_{t} \mid U^{i}, \tau^{i}\right)=\mathbb{E}\left(W_{\tau_{i}} \mid U^{i}, \tau^{i}\right), t \in\left[\tau_{i}, \tau_{i+1}\right) .
$$

Together, the encoding and the decoding policies must satisfy the long-term MSE constraint in (3).

The decoding policy in (6) forces the estimate $\hat{W}_{t}$ to be equal to the conditional expectation of $W_{t}$ given all the received information, which is constant between two consecutive codeword-generating time stamps. Allowing more

\footnotetext{
${ }^{2}$ We refer to encoding and decoding policies to emphasize their causal nature.

${ }^{3}$ In some scenarios, we allow randomness in the mapping $\mathrm{f}_{i}$, replacing the deterministic mapping $\mathrm{f}_{i}$ in (5) by a transition probability kernel.
}

freedom in the design of a decoding policy cannot yield a lower MSE because (6) is the MMSE estimator of $W_{t}$ during $t \in\left[\tau_{i}, \tau_{i+1}\right)$. This is a consequence of the zero-delay MSE constraint (3) at the decoder. As we explain in Section V-B below, had we allowed delay at the decoder, we could have improved performance by e.g. using linear interpolation between recovered samples at the decoder.

\section{B. Operational distortion-rate function}

We now define the operational distortion-rate function.

Definition 3. (Operational distortion-rate function (ODRF)) The ODRF is the minimum distortion compatible with rate $R$ achievable by causal rate- $R$ codes in the limit of infinite time horizon:

$$
D^{\mathrm{op}}(R) \triangleq \limsup _{T \rightarrow \infty} \inf \{d: \exists(R, d, T) \text { causal code }\} .
$$

It turns out that the ODRF can be decomposed into the distortion due to sampling and the distortion due to quantization.

Proposition 1. The ODRF for the Wiener process can be written as

$$
\begin{aligned}
D^{\mathrm{op}}(R)= & \limsup _{T \rightarrow \infty} \inf _{\pi_{T} \in \Pi_{T}} \frac{1}{T}\left\{\mathbb{E}\left(\sum_{i=0}^{N} \int_{\tau_{i}}^{\tau_{i+1}}\left(W_{t}-W_{\tau_{i}}\right)^{2} d t\right)\right. \\
& \left.+\inf _{\substack{f_{T} \in F_{T}: \\
(2)}} \mathbb{E}\left(\sum_{i=1}^{N}\left(\tau_{i+1}-\tau_{i}\right)\left(W_{\tau_{i}}-\hat{W}_{\tau_{i}}\right)^{2}\right)\right\},
\end{aligned}
$$

where $\tau_{N+1} \triangleq T$, and $\Pi_{T}, F_{T}$ denote the sets of all sampling and all compressing policies over the time horizon $T$ respectively.

Furthermore, if randomized compressing policies are allowed, there is no loss of optimality if at time $\tau_{i}$, a compressing policy only takes into account the innovation $W_{\tau_{i}}-\hat{W}_{\tau_{i-1}}$, past codewords $U^{i-1}$ and timing information $\tau^{i}$, rather than the whole process up to time $\tau_{i}$, as permitted by Definition 2 .

\section{Proof. Appendix A.}

In (8a), $W_{\tau_{i}}$ is the MMSE estimator of $W_{t}$ at $t \in\left[\tau_{i}, \tau_{i+1}\right)$, given the past lossless samples $\left\{W_{\tau_{j}}\right\}_{j=1}^{i}$ and the codewordgenerating time stamps $\tau^{i}$. The expectation in (8a) is the sampling distortion due to causally estimating the Wiener process from its lossless samples $\left\{W_{\tau_{j}}\right\}_{j=1}^{i}$ taken under the sampling policy $\pi_{T}$.

The expectation in (8b) is the mean-square quantization error of the samples, accumulated over sampling intervals of length $\tau_{i+1}-\tau_{i}, i=1, \ldots, N$. According to the compressing policy described in Proposition 1, the minimization problem in $(8 \mathrm{~b})$ is the operational zero-delay causal distortion-rate function of the discrete-time stochastic process formed by the samples. Furthermore, the encoding policy can be implemented as a sampler followed by a compressor. See Fig. 2 . The sampler takes measurements of the Wiener process under a sampling policy and outputs samples without delay to the 


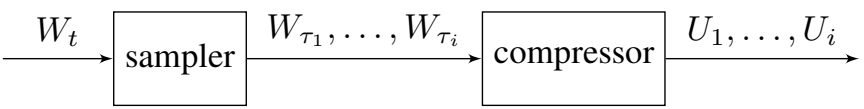

Fig. 2: Decomposition of the encoder.

compressor. Upon receiving a new sample, the compressor immediately generates a codeword under the compressing policy described in Proposition 1.

\section{Informational distortion-rate function}

The directed information $I\left(X^{n} \rightarrow Y^{n}\right)$ from a sequence $X^{n}$ to a sequence $Y^{n}$ is defined as [32]

$$
I\left(X^{n} \rightarrow Y^{n}\right)=\sum_{i=1}^{n} I\left(X^{i} ; Y_{i} \mid Y^{i-1}\right) .
$$

The directed information captures the information due to the causal dependence of $Y^{n}$ on $X^{n}$.

A sampling policy $\pi_{T}=\left\{\tau_{1}, \tau_{2}, \ldots\right\}$ is deterministic if its sampling time stamps (1) are deterministic. We denote the set of all deterministic sampling policies by $\Pi_{T}^{\mathrm{DET}}$. Under a deterministic sampling policy, the total number of samples $N$ within the time horizon $[0, T]$ is constant.

Definition 4. (Informational distortion-rate function (IDRF)) The IDRF for the Wiener process under deterministic sampling policies can be written as

$$
\begin{aligned}
& D_{\mathrm{DET}}(R) \triangleq \\
& \limsup _{T \rightarrow \infty} \inf _{\pi_{T} \in \Pi_{T}^{\mathrm{DET}}} \frac{1}{T}\left\{\mathbb{E}\left(\sum_{i=0}^{N} \int_{\tau_{i}}^{\tau_{i+1}}\left(W_{t}-W_{\tau_{i}}\right)^{2} d t\right)+\right.
\end{aligned}
$$

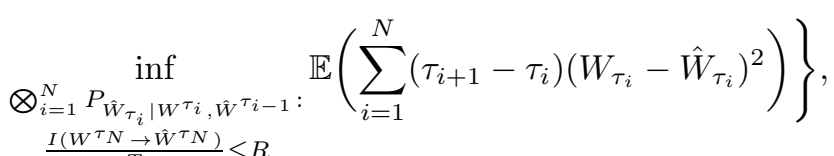

The minimization problem (10b) in $D_{\mathrm{DET}}(R)$ is the causal IDRF for the discrete-time stochastic process formed by the samples. Note that (10b) is minimized over the directed information rate, which gives an information-theoretic lower bound to the rate considered in (2). Thus, the following relation holds according to [36, Eq. (43)].

$$
D_{\mathrm{DET}}^{\mathrm{op}}(R) \geq D_{\mathrm{DET}}(R),
$$

where $D_{\mathrm{DET}}^{\mathrm{op}}(R)$ is the ODRF for deterministic sampling policies defined by (8) with the minimization constraint in (8a) replaced by $\pi_{T} \in \Pi_{T}^{\mathrm{DET}}$.

D. Operational and informational distortion-frequency-rate function

According to Proposition 1, an optimal encoder can be implemented as a sampler followed by a compressor. To gain insight into the tradeoffs between the sampling frequency $f$ at the sampler and the rate per sample $R_{s}$ at the compressor, we define an $\left(f, R_{s}, d, T\right)$ causal code.
Definition 5. (An $\left(f, R_{s}, d, T\right)$ causal code) An $\left(f, R_{s}, d, T\right)$ causal code for the Wiener process $\left\{W_{t}\right\}_{t=0}^{T}$ is a triplet of causal sampling, compressing and decoding policies:

(i) the causal sampling policy ${ }^{4} \pi_{T}=\left\{\tau_{1}, \tau_{2}, \ldots\right\}$ satisfies the average sampling frequency constraint

$$
\frac{1}{T} \mathbb{E}(N)=f
$$

(ii) the compressing policy $f_{T}=\left\{f_{1}, f_{2}, \ldots\right\}^{5}$ is

$$
\mathrm{f}_{i}: \mathbb{R} \times \mathbb{R}^{i-1} \times \mathbb{R}^{i} \rightarrow\left[2^{\ell_{i}}\right] .
$$

The codeword generated at time $\tau_{i}$ is $U_{i}=\mathrm{f}_{i}\left(W_{\tau_{i}}, U^{i-1}, \tau^{i}\right)$. The codewords' lengths must satisfy

$$
\frac{1}{\mathbb{E}(N)} \mathbb{E}\left(\sum_{i=1}^{N} \ell_{i}\right) \leq R_{s} \text { (bits per sample); }
$$

(iii) the decoding policy causally maps the received codewords and the codeword-generating time stamps to a continuous-time process estimate $\left\{\hat{W}_{t}\right\}_{t=0}^{T}$ using (6).

Together, the causal sampling, compressing and decoding policies must satisfy the long-term MSE constraint in (3).

We define the operational distortion-frequency-rate function.

Definition 6. (Operational distortion-frequency-rate function $(O D F R F))$ The ODFRF is the minimum distortion achievable by causal frequency- $f$ and rate- $R_{s}$ codes in the limit of infinite time horizon:

$$
D^{\mathrm{op}}\left(f, R_{s}\right) \triangleq \limsup _{T \rightarrow \infty} \inf \left\{d: \exists\left(f, R_{s}, d, T\right) \text { causal code }\right\} .
$$

Using the method used to decompose $D^{\mathrm{op}}(R)$ in Proposition 1 , we can write $D^{\mathrm{op}}\left(f, R_{s}\right)$ as

$$
\begin{aligned}
& D^{\mathrm{op}}\left(f, R_{s}\right)= \\
& \limsup _{T \rightarrow \infty} \inf _{\substack{\pi_{T} \in \Pi_{T} \\
(12)}} \frac{1}{T}\left\{\mathbb{E}\left(\sum_{i=0}^{N} \int_{\tau_{i}}^{\tau_{i+1}}\left(W_{t}-W_{\tau_{i}}\right)^{2} d t\right)\right. \\
& \left.+\inf _{\substack{f_{T} \in F_{T}: \\
(14)}} \mathbb{E}\left(\sum_{i=1}^{N}\left(\tau_{i+1}-\tau_{i}\right)\left(W_{\tau_{i}}-\hat{W}_{\tau_{i}}\right)^{2}\right)\right\},
\end{aligned}
$$

where the expectation in (16a) is the sampling distortion, and the expectation in (16b) is the mean-square quantization error of the samples weighted by the lengths of sampling intervals $\tau_{i+1}-\tau_{i}, i=1, \ldots, N$.

We define the informational distortion-frequency-rate function for deterministic sampling policies. The informational equivalent of $D^{\mathrm{op}}\left(f, R_{s}\right)$ replaces (14) by the constraint on the directed information, that is, for deterministic sampling policies,

$$
\frac{1}{N} I\left(W^{\tau_{N}} \rightarrow \hat{W}^{\tau_{N}}\right) \leq R_{s} .
$$

\footnotetext{
${ }^{4}$ The causal sampling policy is defined in Definition 2(i)

${ }^{5}$ Here we slightly abuse the notation: we have used $f_{T}$ in Definition 2(ii), and have shown in Proposition 1 that the compressing policy $f_{T}$ can be simplified to (13).
} 
Definition 7. (Informational distortion-frequency-rate function (IDFRF)) The IDFRF for the Wiener process under deterministic sampling policies can be written as

$$
\begin{aligned}
& D_{\mathrm{DET}}\left(f, R_{s}\right) \triangleq \\
& \limsup _{T \rightarrow \infty} \inf _{\substack{\pi_{T} \in \Pi_{T}^{\mathrm{DET}}:(12) \\
(18 \mathrm{a})}} \frac{1}{T}\left\{\mathbb{E}\left(\sum_{i=0}^{N} \int_{\tau_{i}}^{\tau_{i+1}}\left(W_{t}-W_{\tau_{i}}\right)^{2} d t\right)\right. \\
& \left.+\inf _{\substack{\bigotimes_{i=1}^{N} P_{\hat{W}_{\tau_{i}} \mid W^{\tau_{i}}, \hat{W}^{\tau_{i-1}}}: \\
(17)}} \mathbb{E}\left(\sum_{i=1}^{N}\left(\tau_{i+1}-\tau_{i}\right)\left(W_{\tau_{i}}-\hat{W}_{\tau_{i}}\right)^{2}\right)\right\}
\end{aligned}
$$

Similar to $D_{\mathrm{DET}}(R)$ in Definition 3, (18b) is the IDRF for the Gauss-Markov process formed by the samples, but it is worth noticing that the rate considered in (18b) is the rate per sample $R_{s}$ rather than the rate per second $R$ considered in (10b).

\section{Main Results}

The first theorem of this section shows the optimal causal sampling and compressing policies that achieve $D^{\mathrm{op}}(R)$.

Theorem 1. In causal coding of the Wiener process, the optimal causal sampling policy is the following symmetric threshold sampling policy:

$$
\tau_{i+1}=\inf \left\{t \geq \tau_{i}:\left|W_{t}-W_{\tau_{i}}\right| \geq \sqrt{\frac{1}{R}}\right\}, i=0,1,2, \ldots
$$

The optimal compressing policy is a 1-bit sign-of-innovation (SOI) compressor:

$$
U_{i}= \begin{cases}1 & \text { if } \quad W_{\tau_{i+1}}-W_{\tau_{i}} \geq 0 \\ 0 & \text { if } \quad W_{\tau_{i+1}}-W_{\tau_{i}}<0 .\end{cases}
$$

The SOI coding scheme achieves the ODRF:

$$
D^{\mathrm{op}}(R)=\frac{1}{6 R} .
$$

Proof. Section IV-A.

Together with the optimal encoding policy in Theorem 1, the optimal decoding policy (6) accumulates the received noiseless innovations to estimate the current value of the process.

The next theorem shows the optimal deterministic sampling policy that achieves $D_{\mathrm{DET}}(R)$.

Theorem 2. In causal coding of the Wiener process, the uniform sampling with the sampling interval equal to

$$
\tau_{i+1}-\tau_{i}=\frac{1}{R}, i=0,1,2, \ldots,
$$

achieves

$$
D_{\mathrm{DET}}(R)=\frac{5}{6 R} .
$$

Proof. Section IV-D.
Theorem 3. In causal coding of the Wiener process, the ODRF satisfies

$$
\begin{aligned}
D^{\mathrm{op}}(R) & =\min _{\substack{f>0, R_{s} \geq 1: \\
f R_{s} \leq R}} D^{\mathrm{op}}\left(f, R_{s}\right), \\
& =D^{\mathrm{op}}(R, 1),
\end{aligned}
$$

and the IDRF under deterministic sampling policies satisfies

$$
\begin{aligned}
D_{\mathrm{DET}}(R) & =\min _{\substack{f>0, R_{s} \geq 1: \\
f R_{s} \leq R}} D_{\mathrm{DET}}\left(f, R_{s}\right) \\
& =D_{\mathrm{DET}}(R, 1) .
\end{aligned}
$$

Proof. See Section IV-B for the proof of (24). See Section IV-C for the proof of (25).

Using Theorem 3, we can formulate the working principle of an optimal encoding policy as follows. A sampler takes measurements of the Wiener process as fast as possible subject to a rate constraint, and the most recent sample is used to generate a 1-bit codeword, which is transmitted to the decoder without delay. In the setting of Theorem 1, the 1-bit SOI compressor associated with the symmetric threshold sampling policy uses the most recent sample to calculate the innovation and to produce a 1-bit codeword. In the setting of Theorem 2, although evaluating $D_{\mathrm{DET}}(R)$ does not give us an operational compressing policy, we know that the stochastic kernel that achieves the causal IDRF for discrete-time Gauss-Markov processes formed by the samples under uniform sampling policies has the form $\bigotimes_{i=1}^{\infty} P_{\hat{W}_{\tau_{i}} \mid W_{\tau_{i}}-\hat{W}_{\tau_{i-1}}, \hat{W}_{\tau_{i-1}}}$ [35, Eq. (5.12)], suggesting that at the encoder, it is sufficient to compress the quantization innovation $W_{\tau_{i}}-\hat{W}_{\tau_{i-1}}$ only. The decoder computes the estimate $\hat{W}_{\tau_{i}}$ as $\hat{W}_{\tau_{i}}=\hat{W}_{\tau_{i-1}}+$ $\mathrm{q}_{i}\left(W_{\tau_{i}}-\hat{W}_{\tau_{i-1}}\right)$, where $\mathrm{q}_{i}=\mathrm{g}_{i} \circ \mathrm{f}_{i}, \mathrm{f}_{i}\left(W_{\tau_{i}}-\hat{W}_{\tau_{i-1}}\right) \in\left[2^{\ell_{i}}\right]$ is the $i$-th binary codeword, and $\mathrm{g}_{i}(c) \in \mathbb{R}$ is the quantization representation point corresponding to $c \in\left[2^{\ell_{i}}\right]$. In practice, one can use the greedy Lloyd-Max compressor [18] that runs the Lloyd-Max algorithm for the quantization innovation in each step based on the prior probability of the quantization innovation. Specifically, the prior for $(i+1)$-th step is the pdf of the quantization innovation $W_{\tau_{i+1}}-\hat{W}_{\tau_{i}}$, which can be computed as the convolution of the pdfs of the quantization error $W_{\tau_{i}}-\hat{W}_{\tau_{i}}$ and the process increment $W_{\tau_{i+1}}-W_{\tau_{i}}$. The globally optimal scheme has a negligible gain over the greedy Lloyd-Max algorithm even in the finite time horizon [18].

Fig. 3 displays distortion-rate tradeoffs obtained in Theorems 1 and 2, as well as a numerical simulation of the uniform sampling in Theorem 2 with the greedy LloydMax quantization of innovations. The symmetric threshold sampling policy followed by a 1-bit SOI compressor leads to a much lower MSE than uniform sampling. Indeed, according to Theorems 1 and $2, \frac{D_{\mathrm{DET}}(R)}{D^{\mathrm{op}}(R)}=5$, and $D_{\mathrm{DET}}^{\mathrm{op}}(R)$ for the uniform sampling is even higher than $D_{\mathrm{DET}}(R)$ by (11). Note that the greedy Lloyd-Max curve is rather close to the $D_{\text {DET }}(R)$ curve, indicating that the IDRF is a meaningful gauge of what is attainable in zero-delay continuous-time causal compression. 


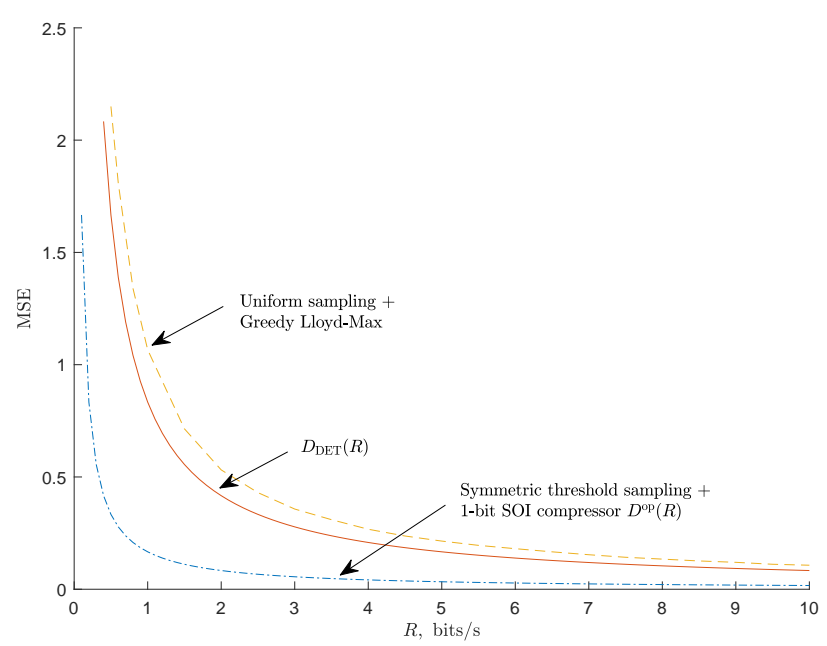

Fig. 3: MSE versus rate

The optimal sampling policies of Theorems 1 and 2, i.e. the symmetric threshold and the uniform sampling policies, are the same as the corresponding optimal sampling policies that achieve the minimum sampling distortion [6, Sec. 3.1] [7] subject to an average sampling frequency constraint (12) with $f=R$. The value of $D^{\mathrm{op}}(R)$ (21) achieved by the symmetric threshold sampling policy is the same as the sampling distortion, since the 1-bit SOI compressor is able to compress each innovation noiselessly due to the size- 2 alphabet of the innovations, resulting in zero quantization distortion (8b). In contrast, for deterministic sampling policies, quantization distortion is unavoidable, since the samples are Gaussian. If we only consider the constraint on the sampling frequency, the optimal deterministic sampling policy for the Wiener process is uniform sampling [6, Sec. 3.1]. Nevertheless, the result in Theorem 2 implies that uniform sampling is still optimal in the IDRF sense, whether or not the quantization distortion is taken into account.

\section{Proof of the Main Results}

\section{A. Proof of Theorem 1}

We first show that the sampling and compressing policies in Theorem 1 achieve the ODRF in (21) (achievability). Then, we show there does not exist other sampling and compressing policies that lead to a lower MSE (converse).

(i) Achievability: The samples taken under the symmetric threshold sampling policy in (19) can be written as

$$
W_{\tau_{i+1}}=W_{\tau_{i}}+\Delta W_{i}, i=0,1,2, \cdots,
$$

where $\Delta W_{i}$ has an alphabet $\left\{ \pm \sqrt{\frac{1}{R}}\right\}$ of size 2. Since the compressor knows $W_{0}=0, \Delta W_{i}$ can be encoded losslessly with 1 bit per sample. Thus, using the 1-bit SOI compressor in (20), the quantization MSE (8b) is zero.

Now the ODRF function only contains the sampling distortion, which is calculated to be $\frac{1}{6 R}$ in [7, Eq. (21)]. We proceed to show that the symmetric threshold policy together with the 1-bit SOI compressor satisfies (2). Under the symmetric threshold sampling policy in (19), $\tau_{i+1}-\tau_{i}$ 's are i.i.d. random variables. The mean of $\tau_{i+1}-\tau_{i}$ is calculated as [30, Thm. 8.5.5]

$$
\mathbb{E}\left(\tau_{i+1}-\tau_{i}\right)=\frac{1}{R}, i=1,2, \ldots,
$$

thus

$$
\mathbb{E}\left(\tau_{N}\right)=\frac{\mathbb{E}(N)}{R} .
$$

Consequently, we verify that (2) is satisfied:

$$
\frac{\mathbb{E}\left(\sum_{i=1}^{N} \ell_{i}\right)}{T}=\frac{\mathbb{E}(N)}{T} \leq \frac{\mathbb{E}(N)}{\mathbb{E}\left(\tau_{N}\right)}=R .
$$

We conclude that $D^{\mathrm{op}}(R) \leq \frac{1}{6 R}$.

(ii) Converse: Since

$$
\mathbb{E}(N) \leq \mathbb{E}\left(\sum_{i=1}^{N} \ell_{i}\right),
$$

the ODRF is lower-bounded by the sampling distortion (8a) subject to the maximum sampling frequency constraint (12) with $f \leq R$, which is equal to $\frac{1}{6 R}$, and is achievable by a symmetric threshold policy with the threshold $\sqrt{\frac{1}{R}}$ [7, Eq. (20)].

\section{B. Proof of Theorem 3 (24)}

$D^{\mathrm{op}}\left(f, R_{s}\right)$ is lower-bounded by the sampling distortion (16a). This lower bound is achieved by a symmetric threshold sampling policy with thresholds $\pm \sqrt{\frac{1}{f}}$, and a 1-bit SOI compressor, where the symmetric threshold sampler achieves the minimum of (16a), which is equal to $\frac{1}{6 f}$ [7, Eq. (20)]. Since the 1-bit SOI compressor results in zero quantization distortion (16b),

$$
D^{\mathrm{op}}\left(f, R_{s}\right)=\frac{1}{6 f},
$$

for any $R_{s} \geq 1$. Plugging (31) into the minimization problem in (24a), we obtain

$$
\begin{gathered}
\min _{\substack{f>0, R_{s} \geq 1: \\
f R_{s} \leq R}} D^{\mathrm{op}}\left(f, R_{s}\right)=D^{\mathrm{op}}(R, 1), \\
D^{\mathrm{op}}(R, 1)=\frac{1}{6 R} .
\end{gathered}
$$

Comparing (32) to (21), we conclude that (24) holds.

\section{Proof of Theorem 3 (25)}

Since the samples taken under a deterministic sampling policy form a Gauss-Markov process, we first compute $D_{\text {DET }}\left(f, R_{s}\right)$ building on existing results on the causal IDRF (18b) of discrete-time Gauss-Markov processes.

Lemma 1. The IDFRF under deterministic sampling policies can be written as

$$
\begin{aligned}
& D_{\mathrm{DET}}\left(f, R_{s}\right)=\limsup _{N \rightarrow \infty} D_{N}\left(f, R_{s}\right), \\
& D_{N}\left(f, R_{s}\right)=\inf _{T^{N} \geq 0:} \frac{f}{N}\left(\sum_{i=0}^{N} \frac{T_{i}^{2}}{2}+\min _{\substack{D^{N} \geq 0: \\
(35)}} \sum_{i=1}^{N} T_{i} D_{i}\right),
\end{aligned}
$$


where the minimization constraints in (33) are

$$
\frac{1}{N} \sum_{i=0}^{N} T_{i}=\frac{1}{f}
$$

and

$$
\begin{aligned}
& z\left(D^{N}\right) \triangleq \frac{1}{N}\left(\sum_{i=1}^{N-1} \log \left(1+\frac{T_{i}}{D_{i}}\right)+\log \left(\frac{T_{0}}{D_{N}}\right)\right) \leq 2 R_{s}, \\
& D_{i-1}+T_{i-1} \geq D_{i}, i=1, \ldots, N .
\end{aligned}
$$

\section{Proof. Appendix B.}

The optimization variable $T^{N}$ in (33) is the vector of sampling intervals $T^{N}=\left\{T_{0}, T_{1}, \ldots, T_{N}\right\}$, where

$$
\begin{aligned}
& T_{i}=\tau_{i+1}-\tau_{i}, i=0, \ldots, N-1, \\
& T_{N}=T-\tau_{N},
\end{aligned}
$$

that determine a deterministic sampling policy. The optimization variable $D^{N}$ in (33) is the vector of sample distortions $D^{N}=\left\{D_{1}, \ldots, D_{N}\right\}$.

Note that $D_{\mathrm{DET}}(R)$ in (10) is related to $D_{\mathrm{DET}}\left(f, R_{s}\right)$ in (33) as follows,

$$
D_{\mathrm{DET}}(R)=\limsup _{N \rightarrow \infty} \inf _{\substack{f>0, R_{s} \geq 1: \\ f R_{s} \leq R}} D_{N}\left(f, R_{s}\right) .
$$

We observe that (25a) does not directly follow (37), since the right-hand side of (25a) switches the order of limsup and inf in (37).

We will use Lemmas 2 and 5 that follow to prove (25a) in Theorem 3.

Lemma 2. $D_{N}\left(f, R_{s}\right)$ is lower-bounded as

$$
\begin{aligned}
& D_{N}\left(f, R_{s}\right) \geq \underline{D}_{N}\left(f, R_{s}\right), \\
& \triangleq \inf _{\substack{T_{0} \geq 0, T_{N} \geq 0 \\
T_{0}+T_{N} \leq \frac{N}{f}}} \frac{f}{2}\left(\frac{T_{0}^{2}+T_{N}^{2}+2 \log e \lambda^{*}\left(f, R_{s}, N\right)}{N}\right. \\
& \left.+\frac{N-1}{N} T^{*}(f, N) \sqrt{T^{*}(f, N)^{2}+4 \log e \lambda^{*}\left(f, R_{s}, N\right)}\right),
\end{aligned}
$$

where $T^{*}(f, N)$ is given by,

$$
T^{*}(f, N) \triangleq \frac{N}{f(N-1)}-\frac{T_{0}+T_{N}}{N-1}, i=1, \ldots, N-1,
$$

and $\lambda^{*}\left(f, R_{s}, N\right) \geq 0$ is the unique solution to

$$
z\left(D^{N *}\right)=2 R_{s}
$$

with $D^{N}$ in (35a) replaced by

$D_{i}^{*}=\frac{-T_{i}+\sqrt{T_{i}^{2}+4 \log e \lambda^{*}\left(f, R_{s}, N\right)}}{2}, i=1, \ldots, N-1$,

$D_{N}^{*}=\frac{\lambda^{*}\left(f, R_{s}, N\right) \log e}{T_{N}}$, and $T_{i}, i=1, \ldots, N-1$ in (35a) replaced by $T^{*}(f, N)$ in (39).

Proof. Appendix C.

Lemma 3. $D_{N}\left(f, R_{s}\right)$ is upper-bounded as

$$
\begin{aligned}
& D_{N}\left(f, R_{s}\right) \leq \bar{D}_{N}\left(f, R_{s}\right), \\
& \triangleq \frac{N}{f(N+1)^{2}}+\frac{\log e \lambda^{*}\left(f, R_{s}, N\right) f}{N} \\
& +\frac{N-1}{2(N+1)} \sqrt{\left(\frac{N}{f(N+1)}\right)^{2}+4 \log e \lambda^{*}\left(f, R_{s}, N\right),}
\end{aligned}
$$

where $\lambda^{*}\left(f, R_{s}, N\right) \geq 0$ is the unique solution to (40) with $D^{N}$ in (35a) replaced by (41) and $T_{i}, i=0, \ldots, N$ in (35a) equal to

$$
T_{0}=T_{1}=\cdots=T_{N}=\frac{N}{f(N+1)} .
$$

Proof. Appendix D.

\section{Lemma 4.}

$$
D_{\mathrm{DET}}\left(f, R_{s}\right)=\frac{1}{2 f}+\frac{1}{f\left(2^{2 R_{s}}-1\right)},
$$

where (44) can be achieved by a uniform sampling policy with sampling intervals equal to

$$
T_{i}=\frac{1}{f}, i=0,1, \ldots
$$

Proof. Appendix E.

\section{Lemma 5.}

$$
D_{\mathrm{DET}}(R)=\min _{\substack{f>0, R_{s} \geq 1: \\ f R_{s} \leq R}} D_{\mathrm{DET}}\left(f, R_{s}\right) .
$$

\section{Proof. Appendix F.}

Using Lemma 5, we conclude that (25a) in Theorem 3 holds. It remains to minimize $D_{\mathrm{DET}}\left(f, R_{s}\right)$ in (25a) over feasible $f$ and $R_{s}$ to prove (25b).

$$
\begin{aligned}
D_{\mathrm{DET}}(R) & =\min _{R_{s} \geq 1} D_{\mathrm{DET}}\left(\frac{R}{R_{s}}, R_{s}\right) \\
& =D_{\mathrm{DET}}(R, 1) \\
& =\frac{1}{2 R}+\frac{1}{3 R}=\frac{5}{6 R},
\end{aligned}
$$

where (47a) holds because $D_{\mathrm{DET}}\left(f, R_{s}\right)$ in (44) decreases monotonically in $f$ for any given $R_{s} \geq 1$, and (47b) holds because $D_{\mathrm{DET}}\left(\frac{R}{R_{s}}, R_{s}\right)$ increases monotonically as $R_{s}$ increases in the range $R_{s} \geq 1$. Thus, $D_{\mathrm{DET}}(R)$ is achieved at $f=R, R_{s}=1$. Note that $\frac{1}{2 R}$ in (47c) comes from the sampling distortion and $\frac{1}{3 R}$ comes from the causal IDRF for the discrete-time samples.

\section{Proof of Theorem 2}

From (47), we conclude that (23) holds. Using Lemma 4 and $(25 \mathrm{~b})$, we conclude that the uniform sampling policy with sampling frequency $R$ achieves $D_{\mathrm{DET}}(R)$. 


\section{RATE-CONSTRAINED SAMPLING WITH DELAYS}

In our communication scenario in Section I-A, the codewords are delivered from the encoder to the decoder without delay, and the distortion constraint (3) penalizes any delay at the encoder or the decoder. While those are realistic assumptions in some scenarios of remote tracking and control, in this section we consider how the achievable distortion-rate tradeoffs are affected if those assumptions are weakened.

\section{A. Delay at the encoder and the decoder}

In the scenario of encoding the entire process for the purpose of preserving it for future, a large delay is permissible. In the extreme, the encoder may wait until the whole input process $\left\{W_{t}\right\}_{t=0}^{T}$ is observed before coding, and the decoder is allowed to wait until $T$ before estimating the process. This corresponds to the classical scenario of non-causal (block) compression. The IDRF for this scenario is given by

$$
\lim _{T \rightarrow \infty} \inf _{\substack{D_{\text {noncausal }}(R)=\\ P_{\left\{\hat{w}_{t}\right\}_{t=0}^{T} \mid\left\{W_{t}\right\}_{t=0}^{T}}^{T} I\left(\left\{W_{t}\right\}_{t=0}^{T} ;\left\{\hat{W}_{t}\right\}_{t=0}^{\bar{T}}\right) \leq R}} \mathbb{E}\left(\frac{1}{T} \int_{0}^{T}\left(W_{t}-\hat{W}_{t}\right)^{2} d t\right)
$$

Berger [34] derived the distortion-rate function for the Wiener process using reverse water-filling over the power spectrum of the process,

$$
D_{\text {noncausal }}(R)=\frac{2 \log _{2} e}{\pi^{2} R} \quad \text { bits/s. }
$$

The ODRF continues to be lower-bounded by the IDRF in this non-causal scenario, $D_{\text {noncausal }}^{\text {op }}(R) \geq D_{\text {noncausal }}(R)$ (cf. (11)). As for the achievability, Berger showed that (49) can be achieved in the following sense: given a rate $R \geq 0$, and $\epsilon>0$, there exists a code with rate $R+\epsilon$ that achieves the distortion $D_{\text {noncausal }}(R)+\epsilon$. Berger's coding scheme operates as follows [34]: the Wiener process is divided into successive time intervals of a large enough length $T$ seconds. For each interval, the Karhunen-Loève (KL) coefficients of the process are calculated, and at most $2^{T(R+\epsilon)}$ codewords are used to jointly encode these coefficients with a resulting MSE per second equal to $D_{\text {noncausal }}(R)+\epsilon$. In parallel with the KL expansion coefficients encoding scheme, an integrating delta modulator is employed to encode each endpoint of the length$T$ intervals with MSE per second $\epsilon$ using $\epsilon$ bits per second.

Comparing $D_{\text {noncausal }}(R)$ in (49) with $D^{\mathrm{op}}(R)$ in (21), we see that, surprisingly, the optimal zero-delay policy outperforms the best infinite delay one:

$$
\frac{D^{\mathrm{op}}(R)}{D_{\text {noncausal }}(R)} \approx 0.57 \text {. }
$$

This is because in zero-delay causal coding, the timing information is free. Indeed, the decoder knows the codewordgenerating time stamps that are stopping times of the filtration generated by the Wiener process. In classical noncausal (block) lossy compression, no encoder and decoder synchronization is assumed, and thus the encoder is tasked with encoding both the values of the Wiener process and the time stamps corresponding to these values. In many operational scenarios of remote tracking and control, the encoder and decoder are naturally synchronized, providing free timing information. Since Berger's distortion-rate function in (49) does not take that into account, it cannot adequately characterize the fundamental information-theoretic limits in those scenarios.

\section{B. Delay at the decoder}

In the scenario of causal coding where some small delay is tolerated but the data is not recorded for storage, e.g. speech communication, one can leverage both the free timing information and the coding delay to improve distortion-rate tradeoffs. A one sample look-ahead decoder waits for the next codeword $U_{\tau_{i+1}}$ before estimating $W_{t}, \tau_{i} \leq t<\tau_{i+1}$, introducing a maximum average delay of $\mathbb{E}\left(\tau_{i+1}-\tau_{i}\right)=\frac{1}{R}$ at the decoder. As we are about to see, this one sample look-ahead decoder greatly reduces the MSE compared to the ODRF obtained in (21) under causal estimation.

With the encoding policy in Proposition 1, the decoder is permitted to estimate $W_{t}$ at time $t^{\prime}, t \leq t^{\prime} \leq T$ using not only the codewords received before time $t$, but also the extra codewords received during the time $\left[t, t^{\prime}\right]$. In the extreme, $t^{\prime}=$ $T$, the decoder can jointly use all the codewords and codewordgenerating time stamps in time horizon $[0, T]$ to recover the Wiener process. Using Wolf and Ziv's decomposition of MSE in [28], the ODRF with decoder delay can be decomposed as

$$
\begin{aligned}
& D_{\text {dec delay }}^{\text {op }}(R)=\limsup _{T \rightarrow \infty} \inf _{\substack{\pi_{T} \in \Pi_{T} \\
f_{T} \in F_{T}:}} \frac{1}{(2)} \mathbb{E}\left(\sum_{i=0}^{N} \int_{\tau_{i}}^{\tau_{i+1}}\left(W_{t}-\bar{W}_{t}\right)^{2}\right. \\
& \left.+\left(\bar{W}_{t}-\hat{W}_{t}\right)^{2} d t\right)
\end{aligned}
$$

where $\bar{W}_{t}$ is the MMSE estimator of the process at the encoder using the samples and the times that they were taken: for $t \in$ $\left[\tau_{i}, \tau_{i+1}\right)$,

$$
\bar{W}_{t} \triangleq \mathbb{E}\left(W_{t} \mid\left\{W_{\tau_{j}}\right\}_{j=1}^{N}, \tau^{N}\right)=\mathbb{E}\left(W_{t} \mid W_{\tau_{i}}, W_{\tau_{i+1}}, \tau_{i}, \tau_{i+1}\right),
$$

where (52) holds because $W_{t}-\left(W_{\tau_{i}}, W_{\tau_{i+1}} \tau_{i}, \tau_{i+1}\right)-$ $\left(\left\{W_{\tau_{j}}\right\}_{j=1}^{i-1},\left\{W_{\tau_{j}}\right\}_{j=i+1}^{N},\left\{\tau_{j}\right\}_{j=1}^{i-1},\left\{\tau_{j}\right\}_{j=i+1}^{N}\right)$ form a Markov chain in that order. Therefore, given all the noiseless samples, $\bar{W}_{t}$ only depends on the previous sample and the next sample. In particular, when the samples are taken under a deterministic sampling policy, $\left(W_{\tau_{i}}, W_{t}, W_{\tau_{i+1}}\right)$ is a Gaussian random vector, thus $\bar{W}_{t}$ in (52) is the linear interpolation between $W_{\tau_{i}}$ and $W_{\tau_{i+1}} . \hat{W}_{t}$ is the MMSE estimator of the process at the decoder using all the received information,

$$
\begin{aligned}
\hat{W}_{t} & =\mathbb{E}\left(W_{t} \mid U^{N}, \tau^{N}\right) \\
& =\mathbb{E}\left(\mathbb{E}\left(W_{t} \mid\left\{W_{\tau_{j}}\right\}_{j=1}^{N}, U^{N}, \tau^{N}\right) \mid U^{N}, \tau^{N}\right) \\
& =\mathbb{E}\left(\bar{W}_{t} \mid U^{N}, \tau^{N}\right),
\end{aligned}
$$

where (53c) holds due to the Markov chain $W_{t}$ $\left(\left\{W_{\tau_{j}}\right\}_{j=1}^{N}, \tau^{N}\right)-U^{N}$ and (52). Since the one sample lookahead decoder only waits until the next codeword $U_{i+1}$ is 
received at $\tau_{i+1}, \hat{W}_{t}$ is specified to $\mathbb{E}\left(\bar{W}_{t} \mid U^{i+1}, \tau^{i+1}\right)$ for $t \in\left[\tau_{i}, \tau_{i+1}\right)$.

We append the one sample look-ahead decoder to the optimal encoding policy in Theorem 1 and calculate the resulting MSE. Under symmetric threshold sampling policies, the samples are not necessarily Gaussian, and the linear interpolation can be suboptimal. Yet, if in (51) we substitute for $\bar{W}_{t}$ a suboptimal estimate $\frac{W_{\tau_{i+1}}+W_{\tau_{i}}}{2}$, then the resulting the MSE is equal to $\frac{1}{12 R}$, a two-fold improvement over (21). We append the one sample look-ahead decoder to the uniform sampling policy in Theorem 2, and ignore the potential reduction in quantization distortion brought by the decoder's ability to look ahead by one sample. The resulting sampling distortion is $\frac{1}{T} \mathbb{E}\left(\sum_{i=0}^{N} \int_{\tau_{i}}^{\tau_{i+1}}\left(W_{t}-\bar{W}_{t}\right)^{2}\right)=\frac{1}{6 R}$, a 3 -fold improvement over the sampling distortion $\frac{1}{2 R}(47 \mathrm{c})$ causally attainable with a uniform sampling policy. Thus, the total MSE is at most $\frac{1}{2 R}$, a 1.67-fold improvement over (23).

\section{Channel delay}

Consider the communication scenario in Fig. 1 with a random channel delay between the codeword-generating time stamp and the codeword-delivery time stamp. The decoder sends an acknowledgement to the encoder once it receives a codeword, and a new codeword is generated only after the previous codeword is delivered. A random delay in the communication channel disrupts the synchronization of timing information, worsening the achievable distortion-rate tradeoffs.

Let $Y_{i}$ be the channel delay. Assume that the initial channel delay is $Y_{0}=0$, and that $0 \leq Y_{i} \leq \tau_{i+1}-\tau_{i}, \forall i=1, \ldots, N$, $\tau_{N+1}=T$ and $Y_{N+1}=0$. The ODRF under the channel delay can be written as

$$
\begin{aligned}
& D_{\text {channel delay }}^{\text {op }}(R) \\
& =\limsup _{T \rightarrow \infty} \inf _{\substack{\pi_{T} \in \Pi_{T} \\
f_{T} \in F_{T}:}} \frac{1}{T} \mathbb{E}\left(\sum_{i=0}^{N} \int_{\tau_{i}+Y_{i}}^{\tau_{i+1}+Y_{i+1}}\left(W_{t}-\hat{W}_{t}\right)^{2} d t\right),
\end{aligned}
$$

where if $t \in\left[\tau_{i}+Y_{i}, \tau_{i+1}+Y_{i+1}\right)$, the optimal decoding policy $\hat{W}_{t}$ is equal to the following MMSE estimator

$$
\hat{W}_{\tau_{i}+Y_{i}} \triangleq \mathbb{E}\left(W_{t} \mid U^{i}, \tau^{i}+Y^{i}\right)=\mathbb{E}\left(W_{\tau_{i}+Y_{i}} \mid U^{i}, \tau^{i}+Y^{i}\right),
$$

and the codeword $U_{i}$ is generated based on the past process $\left\{W_{t}\right\}_{t=0}^{\tau_{i}}$ (Definition 2, (ii)), as in the scenario without the channel delay.

Proposition 2. i) If the delay $Y_{i}$ is independent of the Wiener process, then

$$
D_{\text {channel delay }}^{\mathrm{op}}(R)-D^{\mathrm{op}}(R) \leq \lim _{T \rightarrow \infty} \frac{1}{T R} \mathbb{E}\left(\sum_{i=1}^{N} Y_{i}\right)
$$

where $N$ in (56) is the total number of samples taken under the symmetric threshold sampling policy (19) within the time duration $T$.

ii) If the channel delay $Y_{i}$ is independent of the Wiener process and is i.i.d. distributed as a random variable $Y$, the optimal encoding policy is a symmetric threshold policy with the new threshold $\sqrt{\beta}$ calculated by solving,

$$
\mathbb{E}\left[\max \left(\beta, W_{Y}^{2}\right)\right]=\max \left(\frac{1}{f}, \frac{\mathbb{E}\left[\max \left(\beta^{2}, W_{Y}^{4}\right)\right]}{2 \beta}\right) .
$$

followed by a 1-bit SOI compressor (20). The optimal decoding policy still recovers the samples noiselessly by summing up the received innovations.

Proof. Proposition 2 ii) is proven in the same way as Theorem 1, leveraging the result of Sun et al. [10], who proved that the optimal sampling policy for the Wiener process under a sampling frequency constraint and an i.i.d. channel delay is the symmetric threshold policy with threshold $\sqrt{\beta}$. For the proof of Proposition 2 i), we upper bound $D_{\text {channel delay }}^{\text {op }}(R)$ by the distortion achieved by the SOI coding scheme in Theorem 1. See Appendix G.

\section{CONCLUSION}

The results in this paper contribute to the rich literature on optimal scheduling and causal sequential estimation problems by introducing a transmission rate constraint beyond the popular sampling frequency constraint. The SOI coding scheme is optimal for causal estimation of the Wiener process under an expected rate constraint (Theorem 1). The performance of the SOI coding scheme is much better than that of the best non-causal code (Section V-A). This underscores the power of free information contained in the codeword arrival times that is not considered in the standard setting of noncausal (block) compression. The SOI scheme with a different threshold remains optimal even if the channel introduces an i.i.d. random delay (Proposition 2). The key to transmit information via timing is to use process-dependent, rather than deterministic, sampling time stamps, because the latter contain zero information. The optimal deterministic sampling policy is uniform (Theorem 2). In either setting, the best strategy is to transmit lowest possible rate 1-bit codewords as frequently as possible (Theorem 3). This is a consequence of the real-time distortion constraint (3). If a delay is affordable, the MSE can be further reduced with only one sample look-ahead at the decoder (Section V-B).

\section{REFERENCES}

[1] K. J. Åström and B. M. Bernhardsson, "Comparison of Riemann and Lebesgue sampling for first order stochastic systems," Proceedings of the 41st IEEE Conference on Decision and Control, Las Vegas, NV, USA, pp. 2011-2016 vol.2, Dec. 2002.

[2] O. C. Imer and T. Başar, "Optimal estimation with limited measurements," International Journal of Systems Control and Communications, vol. 2, no. 1-3, pp. 5-29, Jan. 2010.

[3] R. Cogill, S. Lall and J. P. Hespanha, "A Constant Factor Approximation Algorithm for Event-Based Sampling," 2007 American Control Conference, New York, NY, USA, pp. 305-311, 2007.

[4] G. M. Lipsa and N. C. Martins, "Remote state estimation with communication costs for first-order LTI systems," in IEEE Transactions on Automatic Control, vol. 56, no. 9, pp. 2013-2025, Sept. 2011.

[5] J. Wu, Q. Jia, K. H. Johansson and L. Shi, "Event-Based Sensor Data Scheduling: Trade-Off Between Communication Rate and Estimation Quality," in IEEE Transactions on Automatic Control, vol. 58, no. 4, pp. 1041-1046, Apr. 2013. 
[6] M. Rabi and G. V. Moustakides, and J. S. Baras, "Adaptive sampling for linear state estimation," in SIAM Journal on Control and Optimization, vol. 50, no. 2, pp. 672-702, Mar. 2012.

[7] K. Nar and T. Başar, "Sampling multidimensional Wiener processes," 53rd IEEE Conference on Decision and Control, Los Angeles, CA, USA, pp. 3426-3431, Dec. 2014.

[8] J. Chakravorty and A. Mahajan, "Fundamental Limits of Remote Estimation of Autoregressive Markov Processes Under Communication Constraints," in IEEE Transactions on Automatic Control, vol. 62, no. 3, pp. 1109-1124, Mar. 2017.

[9] A. Molin and S. Hirche, "Event-triggered state estimation: An iterative algorithm and optimality properties," in IEEE Transactions on Automatic Control, vol. 62, no. 11, pp. 5939-5946, Nov. 2017.

[10] Y. Sun, Y. Polyanskiy and E. Uysal-Biyikoglu, "Remote estimation of the Wiener process over a channel with random delay," 2017 IEEE International Symposium on Information Theory, Aachen, Germany, pp. 321-325, Jun. 2017

[11] X. Gao, E. Akyol and T. Başar, "Optimal estimation with limited measurements and noisy communication," 2015 54th IEEE Conference on Decision and Control, Osaka, Japan, pp. 1775-1780, Dec. 2015.

[12] X. Ren, J. Wu, K. H. Johansson, G. Shi, and L. Shi, "Infinite horizon optimal transmission power control for remote state estimation over fading channels," in IEEE Transactions on Automatic Control, vol. 63, no. 1, pp. 85-100, Jan 2018.

[13] J. Chakravorty and A. Mahajan, "Remote estimation over a packetdrop channel with Markovian state", in IEEE Transactions on Automatic Control, June 2019.

[14] A. Nayyar, T. Başar, D. Teneketzis, and V. V. Veeravalli, "Optimal strategies for communication and remote estimation with an energy harvesting sensor, IEEE Transactions on Automatic Control, vol. 58, no. 9, pp. 2246-2260, Sept. 2013.

[15] A. Kipnis, A. J. Goldsmith and Y. C. Eldar, "The distortion-rate function of sampled Wiener processes," in IEEE Transactions on Information Theory, vol. 65, no. 1, pp. 482-499, Jan. 2019.

[16] E. Kofman and J. H. Braslavsky, "Level crossing sampling in feedback stabilization under data-rate constraints," in Proceedings of the 45th IEEE Conference on Decision and Control, San Diego, CA, USA, pp. 4423-4428, Dec. 2006.

[17] J. Pearson, J. P. Hespanha and D. Liberzon, "Control With Minimal Cost-Per-Symbol Encoding and Quasi-Optimality of Event-Based Encoders," in IEEE Transactions on Automatic Control, vol. 62, no. 5, pp. 2286-2301, May 2017.

[18] A. Khina, Y. Nakahira, Y. Su and B. Hassibi, "Algorithms for optimal control with fixed-rate feedback," 2017 IEEE 56th Annual Conference on Decision and Control, Melbourne, VIC, Australia, pp. 6015-6020, Dec. 2017.

[19] Q. Ling, "Periodic Event-Triggered Quantization Policy Design for a Scalar LTI System With i.i.d. Feedback Dropouts," in IEEE Transactions on Automatic Control, vol. 64, no. 1, pp. 343-350, Jan. 2019.

[20] M. J. Khojasteh, P. Tallapragada, J. Cortés and M. Franceschetti, "The Value of Timing Information in Event-Triggered Control," in IEEE Transactions on Automatic Control, May 2019.

[21] D. Lehmann and J. Lunze, "Event-based control using quantized state information," IFAC Proceedings Volumes, vol. 43, no. 19, pp.1-6, Sep. 2010.

[22] A. Tanwani, C. Prieurand M. Fiacchini, "Observer-based feedback stabilization of linear systems with event-triggered sampling and dynamic quantization," in Systems and Control Letters, vol. 94, pp. 46-56, Aug. 2016.

[23] M. Abdelrahim, V. S. Dolk and W. P. M. H. Heemels, "Input-tostate stabilizing event-triggered control for linear systems with output quantization," 2016 IEEE 55th Conference on Decision and Control, Las Vegas, NV, USA, pp. 483-488, Dec. 2016.

[24] S. Yoshikawa, K. Kobayashi and Y. Yamashita, "Quantized eventtriggered control of discrete-time linear systems with switching triggering conditions," 2017 56th Annual Conference of the Society of Instrument and Control Engineers of Japan, Kanazawa, pp. 313-316, Sep. 2017.

[25] M. Sun, L. Huang, S. Wang, C. Mao and W. Xie, " Quantized control of event-triggered networked systems with time-varying delays," Journal of the Franklin Institute, May 2018.

[26] P. Tallapragada and J. Cortés, "Event-Triggered Stabilization of Linear Systems Under Bounded Bit Rates," in IEEE Transactions on Automatic Control, vol. 61, no. 6, pp. 1575-1589, Jun. 2016.
[27] P. Tallapragada, M. Franceschetti and J. Cortés, "Event-triggered control under time-varying rates and channel blackouts,"in IFAC Journal of Systems and Control, Aug. 2019.

[28] J. K. Wolf and J. Ziv, "Transmission of noisy information to a noisy receiver with minimum distortion" in IEEE Transactions on Information Theory, vol. 16, no. 4, pp. 406-411, Jul. 1970.

[29] T. Tanaka, K. K. Kim, P. A. Parrilo and S. K. Mitter, "Semidefinite programming approach to Gaussian sequential rate-distortion trade-offs," in IEEE Transactions on Automatic Control, vol. 62, no. 4, pp. 18961910, Apr. 2017.

[30] R. Durrett, Probability: Theory and Examples, 4th ed. Cambridge University Press, 2010.

[31] G. R. Grimmett and D. R. Stirzaker, Probability and Random Processes. Oxford University Press, 2009.

[32] J. Massey, "Causality, feedback and directed information," Proceedings International Symposium on Information Theory and its Applications, pp. 303-305, Nov. 1990. quadratic rate-distortion function for Gaussian stationary sources," in IEEE Transactions on Information Theory, vol. 58, no. 5, pp. 3131-3152, May. 2012.

[33] E. Cinlar, Probability and Stochastics. Springer-Verlag New York, 2014.

[34] T. Berger, "Information rates of Wiener processes," in IEEE Transactions on Information Theory, vol. 16, no. 2, pp. 134-139, Mar. 1970.

[35] P. A. Stavrou, T. Charalambous, C. D. Charalambous, and S. Loyka, "Optimal estimation via nonanticipative rate distortion function and applications to time-varying Gauss-Markov processes," SIAM Journal on Control and Optimization, pp. 3731-3765, Oct. 2018.

[36] V. Kostina and B. Hassibi, "Rate-cost tradeoffs in control," in IEEE Transactions on Automatic Control, Apr. 2019.

[37] A. Braides, $\Gamma$-convergence for beginners. Oxford University Press, 2002.

[38] N. Guo and V. Kostina, "Optimal rate-constrained sampling of the Wiener process", in Proceedings 57th Annual Allerton Conference on Communication, Control and Computing, Monticello, IL, Sep. 2019, to appear.

\section{APPENDIX}

\section{A. Proof of Proposition 1}

Using Definition 2 and 3, the ODRF can be expressed as

$$
D^{\mathrm{op}}(R)=\limsup _{T \rightarrow \infty} \inf _{\substack{\pi_{T} \in \Pi_{T} \\ f_{T} \in F_{T}:}} \frac{1}{T} \mathbb{E}\left(\int_{t=0}^{T}\left(W_{t}-\hat{W}_{t}\right)^{2} d t\right),
$$

where $\hat{W}_{t}$ satisfies (6). The objective function in (58) decomposes in the following way.

$$
\begin{aligned}
& \frac{1}{T} \mathbb{E}\left(\int_{t=0}^{T}\left(W_{t}-\hat{W}_{t}\right)^{2} d t\right) \\
= & \frac{1}{T} \mathbb{E}\left(\sum_{i=0}^{N} \int_{\tau_{i}}^{\tau_{i+1}}\left(W_{t}-\hat{W}_{\tau_{i}}\right)^{2} d t\right) \\
= & \frac{1}{T} \mathbb{E}\left(\sum_{i=0}^{N} \int_{\tau_{i}}^{\tau_{i+1}}\left(W_{t}-W_{\tau_{i}}\right)^{2}\right)+ \\
& \frac{1}{T} \mathbb{E}\left(\sum_{i=0}^{N}\left(\tau_{i+1}-\tau_{i}\right)\left(W_{\tau_{i}}-\hat{W}_{\tau_{i}}\right)^{2} d t\right)+ \\
& \frac{1}{T} \mathbb{E}\left(\sum_{i=0}^{N}\left(W_{\tau_{i}}-\hat{W}_{\tau_{i}}\right) \int_{\tau_{i}}^{\tau_{i+1}}\left(W_{t}-W_{\tau_{i}}\right) d t\right) \\
= & \frac{1}{T} \mathbb{E}\left(\sum_{i=0}^{N} \int_{\tau_{i}}^{\tau_{i+1}}\left(W_{t}-W_{\tau_{i}}\right)^{2}\right)+ \\
& \frac{1}{T} \mathbb{E}\left(\sum_{i=0}^{N}\left(\tau_{i+1}-\tau_{i}\right)\left(W_{\tau_{i}}-\hat{W}_{\tau_{i}}\right)^{2} d t\right),
\end{aligned}
$$


where $(59 \mathrm{~b})$ holds because $\hat{W}_{t}$ is constant in $\left[\tau_{i}, \tau_{i+1}\right), i=$ $1, \ldots, N,(59 \mathrm{c})$ is obtained by substituting $W_{t}-W_{\tau_{i}}+W_{\tau_{i}}-$ $\hat{W}_{\tau_{i}}$ for the term $W_{t}-\hat{W}_{\tau_{i}}$ in (59b), and (59d) holds due to the fact that $\int_{\tau_{i}}^{\tau_{i+1}}\left(W_{t}-W_{\tau_{i}}\right) d t$ is orthogonal to $W_{\tau_{i}}-\hat{W}_{\tau_{i}}$ for all $i=0,1,2, \ldots, N$. Since the encoder only influences the second term in (59d), we move the minimization over the encoder $f_{T}$ in (58) directly in front of the second term in (59d).

To show that $\mathrm{f}_{i}$ only encodes $W_{\tau_{i}}-\hat{W}_{\tau_{i-1}}$ given $U^{i-1}$ and $\tau^{i}$, we first recall a well-known fact. Consider the following lossy source coding model in Fig. 4, where $X \in \mathcal{X}$ and $Y \in \mathcal{Y}$ are available only at the encoder, $C$ is the common information, $\hat{X} \in \hat{\mathcal{X}}$ is the reproduction. Encoder $P_{U \mid X, Y, C}$ and decoder $P_{\hat{X} \mid U, C}$ aim to achieve a given distortion $d=$ $\mathbb{E}(\mathrm{d}(X, \hat{X}))$, where $\mathrm{d}: \mathcal{X} \times \hat{\mathcal{X}} \rightarrow \mathbb{R}^{+}$is the distortion measure, subject to a constraint on the cardinality of the alphabet $\mathcal{U}$ of $U$. Since

$$
\begin{aligned}
& \mathbb{E}(\mathrm{d}(X, \hat{X}) \mid C=c)=\int_{x \in \mathcal{X}} d P_{X \mid C=c}(x) . \\
& \int_{U \in \mathcal{U}} d P_{U \mid X, C=c}(u) \int_{\hat{x} \in \hat{\mathcal{X}}} d P_{\hat{X} \mid U, C=c}(\hat{x}) \mathrm{d}(x, \hat{x}),
\end{aligned}
$$

the knowledge of side information $Y$ is useless at the encoder, i.e. for any encoder-decoder pair $\left(P_{U \mid X, Y, C}, P_{\hat{X} \mid U, C}\right)$, the pair $\left(P_{U \mid X, C}, P_{\hat{X} \mid U, C}\right)$, where $P_{U \mid X, C}$ is the marginal of $P_{U \mid X, Y, C} P_{Y \mid X, C}$, achieves the same expected distortion.

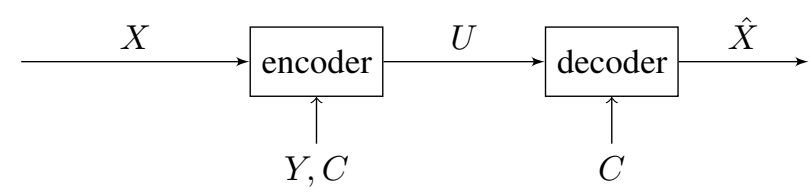

Fig. 4: $Y$ only available at the encoder, $C$ available at both the encoder and the deocder

In our problem, the infimum of the long-term average MSE in (59d) corresponds to a causal sampling policy and a sequence of sample distortion allocations $\mathbb{E}\left(W_{\tau_{i}}-\hat{W}_{\tau_{i}}\right)^{2}$, $i=1,2, \ldots$. At time $\tau_{1}$, we take $X=W_{\tau_{1}}, Y=\left\{W_{t}\right\}_{0<t<\tau_{1}}$, $C=\tau_{1}$, and $d(X, \hat{X})=(X-\hat{X})^{2}$. To achieve a given sample distortion $\mathbb{E}\left(\left(W_{\tau_{1}}-\hat{W}_{\tau_{1}}\right)^{2}\right)$, the random compressing policy needs to only take into account $W_{\tau_{1}}$ and $\tau_{1}$. Inductively, at time $\tau_{i}$, the encoder knows $\left\{W_{t}\right\}_{t=0}^{\tau_{i}}$. Both the encoder and the decoder know $U^{i-1}$ and $\tau^{i}$. Since $\hat{W}_{\tau_{i-1}}$ is known once $U^{i-1}$ and $\tau^{i-1}$ are given,

$$
\begin{aligned}
& \mathbb{E}\left(W_{\tau_{i}}-\hat{W}_{\tau_{i}}\right)^{2}= \\
& \mathbb{E}\left(\left(W_{\tau_{i}}-\hat{W}_{\tau_{i-1}}-\mathbb{E}\left(W_{\tau_{i}}-\hat{W}_{\tau_{i-1}} \mid U^{i}, \tau^{i}\right)\right)^{2}\right) .
\end{aligned}
$$

Take $X=W_{\tau_{i}}-\hat{W}_{\tau_{i-1}}, \hat{X}=\mathbb{E}\left(W_{\tau_{i}}-\hat{W}_{\tau_{i-1}} \mid U^{i}, \tau^{i}\right)$, $U=U_{i}, C=\left\{U^{i-1}, \tau^{i}\right\}$, and $Y$ is everything known at the encoder excluding $X$ and $C$. It follows that for the purpose of achieving the sample distortion $\mathbb{E}\left(W_{\tau_{i}}-\hat{W}_{\tau_{i}}\right)^{2}$, at time $\tau_{i}$, the randomized compressing policy needs to only take into account $W_{\tau_{i}}-\hat{W}_{\tau_{i-1}}, U^{i-1}$, and $\tau^{i}$.

\section{B. Proof of Lemma 1}

Denote the IDRF for discrete-time samples of the Wiener process

$$
W_{\tau_{i+1}}=W_{\tau_{i}}+V_{\tau_{i}}, V_{\tau_{i}} \sim \mathcal{N}\left(0, T_{i}\right)
$$

by $\tilde{D}_{N}\left(R_{s}\right)(18 \mathrm{~b})$. Using the representation of its dual in [29, Eq. (18)] derived using a semi-definite programming approach, we represent $\tilde{D}_{N}\left(R_{s}\right)$ as

$$
\begin{aligned}
& \tilde{D}_{N}\left(R_{s}\right)=\inf _{\substack{D_{i} \geq 0, i=1, \ldots, N: \\
D_{i-1}+T_{i-1} \geq D_{i}, \quad i=1,2, \cdots, N,}} \sum_{i=1}^{N} T_{i} D_{i} . \\
& \frac{1}{N}\left(\sum_{i=1}^{N} \frac{1}{2} \log \left(D_{i-1}+T_{i-1}\right)-\frac{1}{2} \log D_{i}\right) \leq R_{s} .
\end{aligned}
$$

Since the sampling intervals $T^{N}$ are deterministic, we calculate the summand in (18a) as

$$
\mathbb{E}\left(\int_{\tau_{i}}^{\tau_{i+1}}\left(W_{t}-W_{\tau_{i}}\right)^{2} d t\right)=\mathbb{E}\left(\int_{0}^{T_{i}} W_{t}^{2} d t\right)=\frac{T_{i}^{2}}{2} .
$$

Plugging (63) and (64) into (18), we can write $D_{\mathrm{DET}}\left(f, R_{s}\right)$ as

$$
D_{\mathrm{DET}}\left(f, R_{s}\right)=\limsup _{T \rightarrow \infty} \inf _{\substack{\pi_{T} \in \Pi_{T}^{\mathrm{DET}} \\(12)}} \frac{1}{T}\left(\sum_{i=0}^{N} \frac{T_{i}^{2}}{2}+\tilde{D}_{N}\left(R_{s}\right)\right) .
$$

Note that when $T \rightarrow \infty$, the number of samples $N$ must increase no slower than $\sqrt{T}$. Indeed, since the largest sampling interval satisfies

$$
\max _{i=0, \ldots, N} T_{i} \geq \frac{T}{N+1},
$$

the summand in (65)

$$
\frac{\max _{i} T_{i}^{2}}{2 T} \geq \frac{T}{2(N+1)^{2}}
$$

will blow up to infinity if $N$ increases slower than $\sqrt{T}$. Thus, $N \rightarrow \infty$ as $T \rightarrow \infty$. Therefore, we can replace the $\limsup \sup _{T \rightarrow \infty}$ in (65) by $\lim \sup _{N \rightarrow \infty}$ and obtain (33), where we replace $T$ in (65) by $\frac{f}{N}$ as permitted by (12), and we replace the minimization constraint (12) in (65) by its equivalent (34).

\section{Proof of Lemma 2}

We split $D_{N}\left(f, R_{s}\right)$ (33b) into the following optimization problems:

$$
\begin{aligned}
& D_{N}\left(f, R_{s}\right) \triangleq \inf _{\substack{T_{0} \geq 0, T_{N} \geq 0: \\
T_{0}+T_{N} \leq \frac{N}{f}}} D_{N}\left(f, R_{s}, T_{0}, T_{N}\right) \\
& D_{N}\left(f, R_{s}, T_{0}, T_{N}\right) \triangleq \\
& \min _{\substack{T_{1}, \ldots, T_{N-1} \geq 0: \\
\frac{1}{N} \sum_{i=1}^{N-1} T_{i}=\frac{1}{f}-\frac{T_{0}+T_{N}}{N}}} \frac{f}{N}\left(\sum_{i=0}^{N} \frac{T_{i}^{2}}{2}+D_{N}\left(f, R_{s}, T^{N}\right)\right), \\
& D_{N}\left(f, R_{s}, T^{N}\right) \triangleq \min _{\substack{D^{N} \geq 0: \\
(35)}} \sum_{i=1}^{N} T_{i} D_{i} .
\end{aligned}
$$


Denote by $\underline{D}_{N}\left(f, R_{s}, T^{N}\right)$ the lower bound to $D_{N}\left(f, R_{s}, T^{N}\right)$ obtained by deleting the minimization constraint $(35 \mathrm{~b})$ in $(68 \mathrm{c})$, i.e.

$$
\underline{D}_{N}\left(f, R_{s}, T^{N}\right) \triangleq \min _{\substack{D^{N} \geq 0: \\(35 \mathrm{a})}} \sum_{i=1}^{N} T_{i} D_{i}
$$

Denote by $\underline{D}_{N}\left(f, R_{s}, T_{0}, T_{N}\right)$ the corresponding lower bound to $D_{N}\left(f, R_{s}, T_{0}, T_{N}\right)$ in (68b):

$$
\begin{aligned}
& \underline{D}_{N}\left(f, R_{s}, T_{0}, T_{N}\right) \triangleq \\
& \min _{\substack{T_{1}, \ldots, T_{N-1} \geq 0: \\
\frac{1}{N} \sum_{i=1}^{N-1} T_{i}=\frac{1}{f}-\frac{T_{0}+T_{N}}{N}}} \frac{f}{N}\left(\sum_{i=0}^{N} \frac{T_{i}^{2}}{2}+\underline{D}_{N}\left(f, R_{s}, T^{N}\right)\right) .
\end{aligned}
$$

We will calculate the corresponding lower bound to $D_{N}\left(f, R_{s}\right)$ :

$$
\underline{D}_{N}\left(f, R_{s}\right) \triangleq \min _{\substack{T_{0} \geq 0, T_{N} \geq 0: \\ T_{0}+T_{N} \leq \frac{N}{f}}} \underline{D}_{N}\left(f, R_{s}, T_{0}, T_{N}\right) .
$$

We first show that the optimization problem in the right-hand side of (69) is a convex optimization problem that satisfies Slater's condition, i.e. strong duality holds. Then, we solve its Lagrangian dual problem to get the optimal $D_{1}^{*} \ldots, D_{N}^{*}$ in (41) that achieve the minimum in the right-hand side of (69), where $\lambda^{*}\left(f, R_{s}, N\right) \geq 0$ is the unique solution to (40).

The objective function $\sum_{i=1}^{N} T_{i} D_{i}$ (69) is an affine function in $D^{N}$. Furthermore, $z\left(D^{N}\right)$ is a convex function since

$$
\begin{aligned}
& \frac{\partial^{2} z\left(D^{N}\right)}{\partial D_{i}^{2}}=\frac{\log e T_{i}\left(2 D_{i}+T_{i}\right)}{N\left(D_{i}^{2}+D_{i} T_{i}\right)^{2}} \geq 0, \forall i=1, \ldots, N-1, \\
& \frac{\partial^{2} z\left(D^{N}\right)}{\partial D_{N}^{2}}=\frac{\log e}{N D_{N}^{2}} \geq 0 \\
& \frac{\partial^{2} z\left(D^{N}\right)}{\partial D_{i} \partial D_{j}}=0, \forall i, j=1, \ldots, N .
\end{aligned}
$$

Therefore, the minimization problem in the right-hand side of (69) is convex. Notice that $z(D, D, \ldots, D)$ decreases from $+\infty$ to $-\infty$ as $D$ increases from 0 to $\infty$. Thus, there exists $\tilde{D} \geq 0$ such that Slater's condition is satisfied, i.e.

$$
z(\tilde{D}, \tilde{D}, \ldots, \tilde{D})<2 R_{s}
$$

We conclude that 1$)$ the strong duality holds, 2) $\underline{D}\left(f, R_{s}, T^{N}\right)$ can be obtained via its Lagrangian dual problem, and 3) there must exist an optimal Lagrangian multiplier $\lambda^{*}\left(f, R_{s}, N\right) \geq$ 0 that satisfies the complementary slackness (40) in the Karush-Kuhn-Tucker conditions. Indeed, (40) always has a non-negative solution $\lambda^{*}\left(f, R_{s}, N\right)$, since as a function of $\lambda^{*}\left(f, R_{s}, N\right), z\left(D^{N *}\right)$ is continuous and monotonically decreasing from $+\infty$ to $-\infty$ as $\lambda^{*}\left(f, R_{s}, N\right)$ increases from 0 to $+\infty$.
Plugging $D^{N *}$ (41) into (69), we obtain $\underline{D}_{N}\left(f, R_{s}, T^{N}\right)$ and proceed to evaluate $\underline{D}_{N}\left(f, R_{s}, T_{0}, T_{N}\right)$ in (70), which is given by

$$
\underline{D}_{N}\left(f, R_{s}, T_{0}, T_{N}\right)=\min _{\substack{T_{1}, \ldots, T_{N-1} \geq 0: \\ \frac{1}{N} \sum_{i=1}^{N-1} T_{i}=\frac{1}{f}-\frac{T_{0}+T_{N}}{N}}} g\left(T_{1}, \ldots, T_{N-1}\right),
$$

where

$$
\begin{aligned}
& g\left(T_{1}, \ldots, T_{N-1}\right) \triangleq \frac{f}{2 N}\left(T_{0}^{2}+T_{N}^{2}+2 \log e \lambda^{*}\left(f, R_{s}, N\right)\right. \\
& \left.+\sum_{i=1}^{N-1} T_{i} \sqrt{T_{i}^{2}+4 \log e \lambda^{*}\left(f, R_{s}, N\right)}\right) .
\end{aligned}
$$

We make use of the Schur-convexity of (75) to calculate $\underline{D}_{N}\left(f, R_{s}, T_{0}, T_{N}\right)$. Recall that if a function $f\left(x^{d}\right)$ is symmetric and its first partial derivative with respect to each $x_{i}$, $i=1, \ldots, d$ exits, then $f\left(x^{d}\right)$ is Schur-convex if and only if

$$
\left(x_{i}-x_{j}\right)\left(\frac{\partial f\left(x^{d}\right)}{\partial x_{i}}-\frac{\partial f\left(x^{d}\right)}{\partial x_{j}}\right) \geq 0, \forall i, j=1, \ldots, d .
$$

It is clear that $g\left(T_{1}, \ldots, T_{N-1}\right)$ is symmetric since it is invariant to the permutations of $T_{1}, \ldots, T_{N-1}$. To calculate the partial derivatives of (75), we first compute the implicit differentiation $\frac{\partial \lambda^{*}\left(f, R_{s}, N\right)}{\partial T_{i}}$ by taking the derivative with respect to $T_{i}$ on the both sides of (40), yielding

$$
\begin{aligned}
& \frac{\partial \lambda^{*}\left(f, R_{s}, N\right)}{\partial T_{i}}=\frac{1}{\sqrt{T_{i}^{2}+4 \log e \lambda^{*}\left(f, R_{s}, N\right)}} . \\
& \frac{2 \lambda^{*}\left(f, R_{s}, N\right)}{1+\sum_{k=1}^{N-1} \frac{T_{k}}{\sqrt{T_{k}^{2}+4 \log e \lambda^{*}\left(f, R_{s}, N\right)}}} .
\end{aligned}
$$

Using (77) to compute the first partial derivative, we obtain

$$
\begin{aligned}
& \frac{\partial g\left(T_{1}, \ldots, T_{N-1}\right)}{\partial T_{i}} \\
= & \frac{f}{2 N}\left(2 \log e \frac{\partial \lambda^{*}\left(f, R_{s}, N\right)}{\partial T_{i}}+\sqrt{T_{i}^{2}+4 \log e \lambda^{*}\left(f, R_{s}, N\right)}\right. \\
+ & \frac{T_{i}^{2}+2 \log e T_{i} \frac{\partial \lambda^{*}\left(f, R_{s}, N\right)}{\partial T_{i}}}{\sqrt{T_{i}^{2}+4 \log e \lambda^{*}\left(f, R_{s}, N\right)}} \\
+ & \left.\sum_{k=1}^{N} \frac{2 \log e T_{k} \frac{\partial \lambda^{*}\left(f, R_{s}, N\right)}{\partial T_{i}}}{\sqrt{T_{k}^{2}+4 \log e \lambda^{*}\left(f, R_{s}, N\right)}}\right) \\
= & \frac{f}{N} \sqrt{T_{i}^{2}+4 \log e \lambda^{*}\left(f, R_{s}, N\right) .}
\end{aligned}
$$

Using (78), we can verify that $g\left(T_{1}, \ldots, T_{N-1}\right)$ satisfies (76):

$$
\begin{aligned}
& \left(T_{i}-T_{j}\right) \frac{f}{N} \cdot\left(\sqrt{T_{i}^{2}+4 \log e \lambda^{*}\left(f, R_{s}, N\right)}\right. \\
& \left.-\sqrt{T_{j}^{2}+4 \log e \lambda^{*}\left(f, R_{s}, N\right)}\right) \geq 0,
\end{aligned}
$$

for all $i, j=1, \ldots, N-1$. Therefore, $g\left(T_{1}, \ldots, T_{N-1}\right)$ is a Schur-convex function. 
Let $x=\left(x_{1}, \ldots, x_{d}\right) \in \mathbb{R}^{d}, y=\left(y_{1}, \ldots, y_{d}\right) \in \mathbb{R}^{d}$ be two non-increasing sequences of real numbers. Recall that $x$ is majorized by $y$ if for each $k=1, \ldots, d, \sum_{i=1}^{k} x_{i} \leq \sum_{i=1}^{k} y_{i}$ with equality if $k=d$. For a Schur-convex function $f$, if $x$ is majorized by $y$, then $f(x) \leq f(y)$. In our case, the feasible $T_{i}$ 's must satisfy the minimization constraint of the optimization problem in (74). Any sequence $T_{1}, \ldots, T_{N-1}$ that satisfies the minimization constraint of the optimization problem in (74) majorizes the sequence in (39). Therefore, the infimum in (74) is achieved by the sequence $T_{1}^{*}, \ldots, T_{N-1}^{*}$ in (39).

Plugging $T_{1}^{*}, \ldots, T_{N-1}^{*}$ (39) into (74), we obtain

$$
\begin{gathered}
\underline{D}_{N}\left(f, R_{s}, T_{0}, T_{N}\right)=\frac{f}{2}\left(\frac{T_{0}^{2}+T_{N}^{2}+2 \log e \lambda^{*}\left(f, R_{s}, N\right)}{N}\right. \\
\left.+\frac{N-1}{N} T^{*}(f, N) \sqrt{T^{*}(f, N)^{2}+4 \log e \lambda^{*}\left(f, R_{s}, N\right)}\right) .
\end{gathered}
$$

Plugging (80) into the right-hand side of (71) completes the proof.

\section{Proof of Lemma 3}

Plugging (43) into (41), we obtain the corresponding optimal sample distortions,

$$
\begin{aligned}
& D_{1}^{*}=\cdots=D_{N-1}^{*}= \\
& \frac{-\frac{N}{f(N+1)}+\sqrt{\left(\frac{N}{f(N+1)}\right)^{2}+4 \log e \lambda^{*}\left(f, R_{s}, N\right)}}{2}, \\
& D_{N}^{*}=\frac{f(N+1)}{N} \log e \lambda^{*}\left(f, R_{s}, N\right),
\end{aligned}
$$

where $\lambda^{*}\left(f, R_{s}, N\right)$ is defined in Lemma 3.

We first show that the $T^{N}$ in (43) and the corresponding $D^{N}$ in (81) satisfy the deleted constraint (35b), then we can plug $T^{N}$ (43) and $D^{N}$ (81) as feasible solutions into the minimization problem associated with $D_{N}\left(f, R_{s}\right)$ in (33b) to obtain the upper bound in (42).

When $i=2, \ldots, N-1$, the deleted constraint (35b) is satisfied trivially, since $D_{i-1}=D_{i}$ and $T_{i-1} \geq 0$. To prove that the deleted constraint (35b) also holds at $i=1$ and $N$, we upper bound $\lambda^{*}\left(f, R_{s}, N\right)$ for every $N>2$. When

$$
T_{1}=\cdots=T_{N-1},
$$

we can rearrange terms in the complementary slackness condition (40) and conclude $x=\lambda^{*}\left(f, R_{s}, N\right) \log e$ is the unique solution to the following equation,

$$
h_{N}\left(T_{0}, T_{N}, T_{1}, R_{s}, x\right)-x=0,
$$

where

$$
\begin{aligned}
& \quad h_{N}\left(T_{0}, T_{N}, T_{1}, R_{s}, x\right) \triangleq \\
& \quad \frac{T_{1}^{2}}{2^{2 R_{s}+\frac{2}{N-1} R_{s}-\frac{\log T_{0}+\log T_{N}}{N-1}+\frac{\log x}{N-1}}-1} \\
& +\left(\frac{T_{1}}{2^{2 R_{s}+\frac{2}{N-1} R_{s}-\frac{\log T_{0}+\log T_{N}}{N-1}+\frac{\log x}{N-1}}-1}\right)^{2} .
\end{aligned}
$$

Note that the left-hand side of (83) monotonically decreases as $x$ increases.

Given $R_{s}$, plugging (43) into the left-hand side of (83), we conclude that the $\lambda^{*}\left(f, R_{s}, N\right)$ in Lemma 3 is the unique solution to the following equation,

$$
h_{N}\left(\frac{N}{f(N+1)}, \frac{N}{f(N+1)}, \frac{N}{f(N+1)}, R_{s}, x\right)-x=0,
$$

Plugging

$$
x=\frac{N^{2}}{2 f^{2}(N+1)^{2}}
$$

into (85), we observe that the left-hand side of (85) is less or equal to 0 for all $N>2$. Thus, we conclude

$$
\lambda^{*}\left(f, R_{s}, N\right) \log e \leq \frac{N^{2}}{2 f^{2}(N+1)^{2}}, \forall N>2 .
$$

Plugging (87) into (81), we obtain

$$
\begin{aligned}
& D_{1}^{*} \leq \sqrt{\lambda^{*}\left(f, R_{s}, N\right) \log e} \leq \frac{N}{f(N+1)}, \\
& D_{N}^{*} \leq \frac{N}{2 f(N+1)},
\end{aligned}
$$

Substituting (43) and (88) into (35b), we conclude that (35b) holds for $i=1$ and $i=N$.

Now, we can plug (43) and (81) as feasible solutions into (33b) to obtain the right-hand side of (42).

\section{E. Proof of Lemma 4}

From Lemmas 2 and 3, and (33a),

$$
\liminf _{N \rightarrow \infty} \underline{D}_{N}\left(f, R_{s}\right) \leq D_{\mathrm{DET}}\left(f, R_{s}\right) \leq \limsup _{N \rightarrow \infty} \bar{D}_{N}\left(f, R_{s}\right) .
$$

We prove (44) by showing that both bounds are equal to the right-hand side of (44).

To compute the lower bound in (89), we need to understand the behavior of $T^{*}(f, N), \lambda^{*}\left(f, R_{s}, N\right)$ and $T_{0}^{*}, T_{N}^{*}$ as $N$ goes to infinity, where $T_{0}^{*}, T_{N}^{*}$ achieve the minimum of the left-hand side of (89). $T_{0}^{*}$ and $T_{N}^{*}$ must increase as

$$
T_{0}^{*}+T_{N}^{*}=O(\sqrt{N})
$$

or $\frac{T_{0}^{* 2}+T_{N}^{* 2}}{N}$ in (38b) will blow up to infinity as $N \rightarrow \infty$. Substituting (90) to (39), we obtain

$$
T^{*}(f, N)=\frac{1}{f}+O\left(\frac{1}{\sqrt{N}}\right) .
$$

We proceed to compute

$$
\lambda^{*} \triangleq \lim _{N \rightarrow \infty} \lambda^{*}\left(f, R_{s}, N\right) .
$$

For given $T_{0}^{*}, T_{N}^{*}$ and $R_{s}, x=\lambda^{*}\left(f, R_{s}, N\right) \log e$ is the unique solution to (83) with $T_{0}, T_{N}$, and $T(N)$ replaced by $T_{0}^{*}, T_{N}^{*}$ and $T^{*}(f, N)$ in (39). We prove that

$$
\begin{aligned}
& \lambda^{*} \log e \geq \frac{1}{2^{2 R_{s}} f^{2}}, \\
& \lambda^{*} \log e \leq \frac{1}{2 f^{2}} .
\end{aligned}
$$


We substitute (90) and (91) into the left-hand side of (83) and take $\lim _{N \rightarrow \infty}$ to conclude that

$$
\lim _{N \rightarrow \infty} h_{N}\left(T_{0}^{*}, T_{N}^{*}, T^{*}(f, N), R_{s}, \frac{1}{2 f^{2}}\right)-\frac{1}{2 f^{2}} \leq 0 .
$$

Using the fact that the left-hand side of (83) is monotonically decreasing in $x$, we conclude (93a) holds. To prove (93b), we similarly compute

$$
\lim _{N \rightarrow \infty} h_{N}\left(T_{0}^{*}, T_{N}^{*}, T^{*}(f, N), R_{s}, \frac{1}{2^{2 R_{s}} f^{2}}\right)-\frac{1}{2^{2 R_{s}} f^{2}} \geq 0 .
$$

Via the squeeze theorem, (93) implies

$$
\lambda^{*}\left(f, R_{s}, N\right)=O(1) .
$$

Plugging (90), (91) and (96) into (83), and taking $N \rightarrow \infty$ on both sides of (83), we obtain

$$
\lambda^{*} \log e=\frac{1}{f^{2}\left(2^{2 R_{s}}-1\right)^{2}}+\frac{1}{f^{2}\left(2^{2 R_{s}}-1\right)} .
$$

Plugging (90), (91) and (97) into the right-hand side of (38b) and taking $\lim _{N \rightarrow \infty}$, we compute

$$
\begin{aligned}
& \lim _{N \rightarrow \infty} \underline{D}_{N}\left(f, R_{s}\right) \\
& =\frac{1}{2 f}+\frac{1}{f\left(2^{2 R_{s}}-1\right)}+\lim _{\substack{N \rightarrow \infty \\
T_{0} \geq 0, T_{N} \geq 0 \\
T_{0}+T_{N} \leq \frac{N}{f}}} \frac{f}{2}\left(\frac{T_{0}^{2}+T_{N}^{2}}{N}\right) \\
& =\frac{1}{2 f}+\frac{1}{f\left(2^{2 R_{s}}-1\right)},
\end{aligned}
$$

where 0 is achieved in the last term of (98a) by choosing any pair of $T_{0}, T_{N} \geq 0$ that satisfies

$$
T_{0}+T_{N}=o(\sqrt{N}) .
$$

We choose $T_{0}$ and $T_{N}$ in (43) that satisfy (99), such that together with $T_{1}, \ldots, T_{N-1}$ in (43), the lower bound of $D_{\mathrm{DET}}\left(f, R_{s}\right)$ in (89) is achieved.

Now, we compute the upper bound in the right-hand side of (89). $\lambda^{*}\left(f, R_{s}, N\right) \log e$ in (42b) is the unique solution to (83). Note that (97) holds for any $T_{0}$ and $T_{N}$ that satisfy (90). Since $T_{0}$ and $T_{N}$ in (43) satisfy (90), we conclude that the $\lim _{N \rightarrow \infty}$ of $\lambda^{*}\left(f, R_{s}, N\right) \log e$ in (42b) is also equal to (97). Plugging (97) into the right-hand side of (42b) and taking $\lim \sup _{N \rightarrow \infty}$, we calculate that the upper bound of $D_{\mathrm{DET}}\left(f, R_{s}\right)$ in (89) is equal to (98b).

Furthermore, we observe that the uniform sampling intervals (43) achieving both the upper and the lower bound of $D_{\text {DET }}\left(f, R_{s}\right)$, converge to $\frac{1}{f}$ asymptotically. We conclude that the uniform sampling policy with the sampling interval $\frac{1}{f}$ achieves $D_{\mathrm{DET}}\left(f, R_{s}\right)$.

\section{F. Proof of Lemma 5}

The max-min inequality and (37) imply that

$$
D_{\mathrm{DET}}(R) \leq \min _{\substack{f>0, R_{s} \geq 1: \\ f R_{s} \leq R}} \limsup _{N \rightarrow \infty} \bar{D}_{N}\left(f, R_{s}\right) .
$$

On the other hand,

$$
\begin{aligned}
D_{\mathrm{DET}}(R) & \geq \lim _{\substack{N \rightarrow \infty \\
f>0, R_{s} \geq 1:}} \inf _{\substack{f R_{s} \leq R\\
}}\left(f, R_{s}\right) \\
& =\inf _{\substack{f>0, R_{s} \geq 1: N \rightarrow \infty \\
f R_{s} \leq R}} \lim _{N \rightarrow \infty} \underline{D}_{N}\left(f, R_{s}\right),
\end{aligned}
$$

where (101a) is by (37), and (101b) will be proved in the sequel. Using (89) with both bounds equal to each other, (100) and (101), we complete the proof of Lemma 5.

We proceed to prove (101b) via the fundamental theorem of $\Gamma$-convergence. Let $\mathcal{X}$ be a topological space and $G_{N}: \mathcal{X} \rightarrow$ $[0,+\infty], N=1,2, \ldots$, be a sequence of functions defined on $\mathcal{X}$. A sequence of functions $G_{N}, N=1,2, \ldots \Gamma$-converges to its $\Gamma$-limit $G: \mathcal{X} \rightarrow[0,+\infty]$ if [37]:

(i) For every $x \in \mathcal{X}$, and for every sequence $x_{N} \in \mathcal{X}, N=$ $1,2, \ldots$ converging to $x$,

$$
G(x) \leq \liminf _{N \rightarrow \infty} G_{N}\left(x_{N}\right)
$$

(ii) For every $x \in \mathcal{X}$, there exists a sequence $x_{N} \in \mathcal{X}, N=$ $1,2, \ldots$ converging to $x$ such that

$$
G(x) \geq \limsup _{N \rightarrow \infty} G_{N}\left(x_{N}\right)
$$

A sequence of functions $G_{N}, N=1,2, \ldots$ is equicoercive [37] if there exists a compact set $\mathcal{K}$ that is independent of $N$, such that

$$
\inf _{x \in \mathcal{X}} G_{N}(x)=\inf _{x \in \mathcal{K}} G_{N}(x)
$$

The fundamental theorem of $\Gamma$-convergence [37] says that if $G_{N}$ is equicoercive and $\Gamma$-converges to $G: \mathcal{X} \rightarrow[0,+\infty]$, then we have,

$$
\min _{x \in \mathcal{X}} G(x)=\lim _{N \rightarrow \infty} \inf _{x \in \mathcal{X}} G_{N}(x) .
$$

We will show that for any scalars $f>0, R_{s} \geq 1$ and for any sequences $f_{(N)} \rightarrow f, R_{s(N)} \rightarrow R_{s}$, we have

$$
\lim _{N \rightarrow \infty} \underline{D}_{N}\left(f_{(N)}, R_{s(N)}\right)=D_{\mathrm{DET}}\left(f, R_{s}\right)
$$

which means in particular that $D_{\mathrm{DET}}(\cdot, \cdot)$ is the $\Gamma$-limit of $\underline{D}_{N}(\cdot, \cdot)$. We will also prove that $\underline{D}_{N}\left(f, R_{s}\right)$ is equicoercive, and (101b) will follow via the fundamental theorem of $\Gamma$ convergence.

We verify that the reasoning in (90)-(98) goes through replacing $f$ and $R_{s}$ by $f_{(N)}$ and $R_{s(N)}$ respectively, hence (106) holds. 
It remains to prove that $\underline{D}_{N}\left(f, R_{s}\right)$ is equicoercive. Ignoring the two non-negative $\lambda^{*}\left(f, R_{s}, N\right)$ terms in the right-hand side of (38b), we observe that

$$
\begin{aligned}
& \underline{D}_{N}\left(f, R_{s}\right) \\
\geq & \inf _{\substack{T_{0} \geq 0, T_{N} \geq 0 \\
T_{0}+T_{N} \leq \frac{N}{f}}} \frac{f}{2}\left(\frac{T_{0}^{2}+T_{N}^{2}}{N}+\frac{N-1}{N} T^{*}(f, N)^{2}\right) \\
= & \inf _{\substack{T_{0} \geq 0, T_{N} \geq 0 \\
T_{0}+T_{N} \leq \frac{N}{f}}} \frac{1}{2}\left(f \frac{T_{0}^{2}+T_{N}^{2}}{N}\right. \\
+ & \left.\frac{N}{f(N-1)}\left(1-\frac{f\left(T_{0}+T_{N}\right)}{N}\right)^{2}\right),
\end{aligned}
$$

where (107b) is obtained by plugging (39) into (107a). Denote the objective function in (107b) by $q\left(T_{0}, T_{N}\right)$. We prove that $q\left(T_{0}, T_{N}\right)$ is a Schur-convex function: 1) $q\left(T_{0}, T_{N}\right)$ is symmetric, since it is invariant to the permutations of $T_{0}$ and $\left.T_{N} ; 2\right)$ the first-order partial derivatives of $q\left(T_{0}, T_{N}\right)$ with respect to $T_{0}$ and $T_{N}$ are

$$
\begin{aligned}
& \frac{\partial q}{\partial T_{0}}=\frac{f}{N} T_{0}+\frac{f}{N(N-1)}\left(T_{0}+T_{N}\right)-\frac{1}{N-1}, \\
& \frac{\partial q}{\partial T_{N}}=\frac{f}{N} T_{N}+\frac{f}{N(N-1)}\left(T_{0}+T_{N}\right)-\frac{1}{N-1},
\end{aligned}
$$

where (108) satisfies (76). Using the property of Schur-convex functions stated in Lemma 2 after (79), we know that the minimum of $q\left(T_{0}, T_{N}\right)$ is achieved by

$$
T_{0}=T_{N}=a .
$$

for some

$$
0 \leq a \leq \frac{N}{2 f}
$$

Plugging (109) into $q\left(T_{0}, T_{N}\right)$, and minimizing $q(a, a)$ under the constraint (110), we find that the optimal $a$ that minimizes $q(a, a)$ is given by

$$
a=\frac{N}{(N+1) f} .
$$

Plugging (109) and (111) into (107b), we obtain

$$
\underline{D}_{N}\left(f, R_{s}\right) \geq \frac{N^{2}}{2 f(N+1)^{2}} .
$$

On the other hand, plugging (87) into the right-hand side of (38), we obtain

$$
\bar{D}_{N}\left(f, R_{s}\right) \leq \frac{3 N}{2 f(N+1)^{2}}+\frac{\sqrt{3} N(N-1)}{2 f(N+1)^{2}} .
$$

Choosing $f=R$ in (113), we conclude that

$$
\inf _{\substack{f>0, R_{s} \geq 1 \\ f R_{s} \leq R}} \underline{D}_{N}\left(f, R_{s}\right) \leq \frac{3 N}{2 R(N+1)^{2}}+\frac{\sqrt{3} N(N-1)}{2 R(N+1)^{2}} .
$$

For any

$$
f \in\left(0, \frac{R}{3+\sqrt{3}}\right)
$$

the right-hand side of (112) is larger than the right-hand side of (114), thus $f$ in (115) cannot attain the infimum in (114). It follows that the infimum is attaned in the following compact set for $f$,

$$
f \in\left[\frac{R}{3+\sqrt{3}}, R\right],
$$

where the upper bound of $f$ is obtained by lower-bounding $R_{s}$ by 1 . Correspondingly, $R_{s}$ lies within the following compact set,

$$
R_{s} \in[1,3+\sqrt{3}],
$$

Using (116) and (117), we conclude that $\underline{D}_{N}\left(f, R_{s}\right)$ is equicoercive.

\section{G. Proof of Proposition 2 i)}

When the channel delay $Y_{i}$ is independent of the process, and the SOI coding scheme in Theorem 1 is used, the MMSE estimator of the process at time $t \in\left[\tau_{i}+Y_{i}, \tau_{i+1}+Y_{i+1}\right)$ is

$$
\hat{W}_{t}=\mathbb{E}\left(W_{t} \mid U^{i}, \tau^{i}+Y^{i}\right)=W_{\tau_{i}} .
$$

Similar to decomposing $D^{\mathrm{op}}(R)$ in Proposition 1, we decompose the objective function of $D_{\text {channel delay }}^{\text {op }}(R)$ in (54) as

$$
\begin{aligned}
& \frac{1}{T} \mathbb{E}\left(\sum_{i=0}^{N} \int_{\tau_{i}+Y_{i}}^{\tau_{i+1}+Y_{i+1}}\left(W_{t}-W_{\tau_{i}}\right)^{2}+\left(W_{\tau_{i}}-\hat{W}_{\tau_{i}}\right)^{2} d t\right) \\
= & \frac{1}{T} \mathbb{E}\left(\sum_{i=0}^{N} \int_{\tau_{i}+Y_{i}}^{\tau_{i+1}+Y_{i+1}}\left(W_{t}-W_{\tau_{i}}\right)^{2} d t\right),
\end{aligned}
$$

where (119a) holds since $W_{\tau_{i}}-\hat{W}_{\tau_{i}}$ is orthogonal to $\int_{\tau_{i}+Y_{i}}^{\tau_{i}+Y_{i+1}}\left(W_{t}-W_{\tau_{i}}\right) d t$, and $(119 \mathrm{~b})$ holds since the SOI coding scheme can noiselessly recover $W_{\tau_{i}}$. The MSE without delay is simply (119b) with $Y_{1}=\cdots=Y_{N}=0$. Subtracting the MSE without channel delay from the MSE with channel delay, we get the following summation

$$
\frac{1}{T} \mathbb{E}\left(\sum_{i=1}^{N} \int_{\tau_{i}}^{\tau_{i}+Y_{i}}\left(W_{t}-W_{\tau_{i-1}}\right)^{2}-\left(W_{t}-W_{\tau_{i}}\right)^{2} d t\right) .
$$

Substituting $\left(W_{t}-W_{\tau_{i}}+W_{\tau_{i}}-W_{\tau_{i-1}}\right)^{2}$ for the first term $\left(W_{t}-W_{\tau_{i-1}}\right)^{2}$ in (120), and using the fact that $W_{\tau_{i}}-W_{\tau_{i-1}}$ is orthogonal to $\int_{\tau_{i}}^{\tau_{i}+Y_{i}}\left(W_{t}-W_{\tau_{i}}\right) d t$, we further simplify (120) to

$$
\frac{1}{T} \mathbb{E}\left(\sum_{i=1}^{N} Y_{i}\left(W_{\tau_{i}}-W_{\tau_{i-1}}\right)^{2}\right)=\frac{1}{T R} \mathbb{E}\left(\sum_{i=1}^{N} Y_{i}\right),
$$

where the equality in (121) is obtained by plugging in $\left(W_{\tau_{i}}\right.$ $\left.W_{\tau_{i-1}}\right)^{2}=\frac{1}{R}$ under the SOI coding scheme in Theorem 1. Taking $\lim _{T \rightarrow \infty}$ on both sides of (121), we obtain (56). 